La

Révolution

française

\section{La Révolution française}

Cahiers de l'Institut d'histoire de la Révolution française

$3 \mid 2012$

Les comités des assemblées révolutionnaires : des laboratoires de la loi

\title{
La Convention ou l'empire des lois
}

Le Comité de législation et la commission de classification des lois

The Empire of Laws under the Convention. The Legislation committee and the commission for the classification of laws

Annie Jourdan

\section{(2) OpenEdition}

\section{Journals}

Édition électronique

URL : https://journals.openedition.org/lrf/730

DOI : $10.4000 /$ Irf.730

ISSN : 2105-2557

Éditeur

IHMC - Institut d'histoire moderne et contemporaine (UMR 8066)

Référence électronique

Annie Jourdan, «La Convention ou l'empire des lois », La Révolution française [En ligne], 3 | 2012, mis en ligne le 20 décembre 2012, consulté le 21 septembre 2021. URL : http://journals.openedition.org/ Irf/730 ; DOI : https://doi.org/10.4000/Irf.730

Ce document a été généré automatiquement le 21 septembre 2021.

(c) La Révolution française 


\title{
La Convention ou l'empire des lois
}

\author{
Le Comité de législation et la commission de classification des lois \\ The Empire of Laws under the Convention. The Legislation committee and the \\ commission for the classification of laws
}

\section{Annie Jourdan}

1 Et si la période de l'an II était décrite en fonction de l'ensemble des lois qu'elle a promulguées? Car des milliers de lois ont dès lors été conçues, promulguées et plus ou moins fidèlement appliquées durant la période. Une telle approche paraîtrait peut-être irréelle, voire irréaliste. Elle redresserait pourtant un équilibre quelque peu bancal, puisque depuis deux ou trois décennies, la Révolution française est très souvent dépeinte sous sa face sombre, celle des violences et des massacres, comme si elle se résumait exclusivement dans les drames sanglants qui en ont ponctué les séquences ${ }^{1}$. C'est en somme ce dont se plaignait Barère en 1836 dans une lettre à l'historien Léonard Gallois. $\mathrm{Au}$ «noble vengeur de la Convention nationale» qui venait de consacrer un ouvrage à cette assemblée, il rappelait : « dans cette seconde partie contre-révolutionnaire depuis le 9 Thermidor, la terreur royaliste ne vaut pas mieux que la terreur révolutionnaire, mais du moins cette dernière avait pour but la liberté et l'égalité $»^{2}$. Il n'avait pas tort, en ce sens qu'une multitude de lois ont été décrétées en l'an II, qui ne touchent pas seulement à la justice pénale, mais encore à la société civile, aux secours publics et aux institutions républicaines ${ }^{3}$. A la tête de cette entreprise se trouvait notamment le Comité de législation, dont jusqu'à présent on a plutôt sous-estimé le rôle $e^{4}$.

\section{Le Comité de législation}

Baptisé Comité de législation civile et criminelle, cet important organe de la Révolution avait été créé le 13 octobre 1791 par l'Assemblée législative. Le comité avait d'abord été divisé en huit sections afin de préparer un projet de code civil et un code de procédure civile. Il succédait en somme au Comité de législation criminelle établi par la Constituante le 14 septembre 1789, aux côtés du Comité de constitution ${ }^{5}$. Modifié le 22 janvier 1790 avant de devenir durable, il resurgit donc le 13 octobre 1791, chargé désormais de la 
législation civile, et non plus seulement criminelle. Le 9 janvier 1792, le comité est définitivement réorganisé en deux sections comptant chacune vingt-quatre membres. La première prend le nom de « section systématique » et a pour mission de préparer les lois civiles et criminelles; la seconde sous l'appellation « section des rapports » doit étudier les pétitions adressées à la Convention et en faire le rapport aux séances plénières du dit comité. Le 23 janvier suivant, une commission composée de Ducastel, Robin, Guadet et Gohier propose un plan de travail pour la section systématique, qui est aussitôt accepté. Il prévoit de diviser cette section en huit sous-sections de trois membres, chacune chargée d'une partie des réformes à venir. Dans la première, Thorillon, Charlier et Garran-Coulon travailleront sur la procédure civile de première instance et d'appel, de requête civile et de cassation relativement à tous les tribunaux en place. La deuxième section - Corbel, Gohier et Froudière - se voit confier le mariage, les enfants légitimes ou naturels, l'adoption, etc. Dans la troisième, Hua (remplacé par Bigot en mars suivant), Codet et François des Vosges s'occupent des tutelles et des curatelles. Une quatrième section (Azéma, Briolat, Guadet) est chargée des choses meubles et immeubles, des propriétés ; des servitudes et alluvions. La cinquième avec Ducastel, Laplaigne et Lesueur travaille sur la transmission des biens, les donations, testaments, codicilles, fidéicommis, substitutions. Dans la sixième, Delmas, Robin et Bournel s'intéressent aux successions légitimes et aux partages. Aux septième et huitième (Labastide, Caubère, Vimard et Ribbes, Veirieu, et de la Coste) adviennent les obligations, les contrats et les conventions matrimoniales. La législation civile est donc à l'ordre du jour, elle occupe et occupera durablement un grand nombre de députés ${ }^{6}$. De fait, durant la Législative, ce comité s'est soucié non seulement de la présentation des projets de droit constitutionnel, mais aussi de droit civil, d'organisation judiciaire, de droit criminel, de police civile et de police militaire.

3 Sous la Convention, le comité prend le nom de Comité de législation civile, criminelle et de féodalité. Ainsi était supprimé de fait le comité féodal créé par la Constituante. Le 14 octobre 1792, la Convention prend connaissance du nom des quarante-huit membres et des quatorze suppléants qui le composent. Le 10 novembre 1792, ses membres sont répartis entre une section civile et une section criminelle. S'y retrouvent notamment Lanjuinais, Garran-Coulon, Lepelletier et Philippeaux. En décembre, Cambacérès accède à la présidence, tandis que Lanjuinais est élu vice-président et que le secrétariat revient à Azéma et à Robert Lindet. Le 12 janvier 1793, nouveau remaniement. Pour la première fois, Merlin de Douai figure parmi les membres - aux côtés de Buzot, Louvet, Lanjuinais, Larivière, (Robert) Lindet, Mailhe, Saladin et Vergniaud - et bien d'autres encore, moins connus. Le 28 janvier, une nouvelle réorganisation divise le comité en 4 sections de douze membres chacune. Les trois premières sont consacrées à la législation civile ; la quatrième au Code pénal et aux changements à y apporter ${ }^{7}$. Merlin en fait partie, alors que Cambacérès figure dans la deuxième consacrée aux successions et aux enfants nés hors le mariage.

A la suite des journées du 31 mai et du 2 juin, la Convention doit renouveler et compléter tous les comités, exception faite du Comité de salut public qui se maintient tel quel. A l'instigation de Cambacérès qui se plaint de la surcharge de travail et insiste sur l'urgence de donner au peuple "des lois analogues au gouvernement républicain", le Comité de législation est alors divisé en deux sections. L'une composée de douze membres s'occupera de la révision du Code civil et du Code criminel et «d'en approprier les dispositions aux bases du gouvernement républicain $»^{8}$. L'autre section sera chargée des 
rapports sur les affaires particulières qui lui seront renvoyées par la Convention nationale. Le 28 juin suivant, le comité renouvelé se remet au travail'. S'y retrouvent Azéma, Bar, Berlier, Bézard, Cambacérès, Charlier, Delaunay aîné, Florent Guyot, GarranCoulon, Génissieu, Lacoste, Merlin, Oudot, Ricord ${ }^{10}$. Entre-temps, les Girondins ont donc disparu et sont remplacés soit par leurs suppléants, soit par des députés tirés au sort, tandis que s'y perpétuent les deux juristes les plus importants de la Révolution et de l'Empire. Ce sont eux aussi qui très souvent présenteront à la tribune de la Convention les lois concoctées par le dit comité, et ce, au-delà du 9 Thermidor : Cambacérès se chargeait entre-temps de ce qui touchait aux projets du Code civil et Merlin de Douai, plus particulièrement, de ce qui concernait le droit pénal et les pétitions en rapport avec ce droit. A partir du 3 floréal an II, tous deux étaient également impliqués dans le recensement et la classification des lois ${ }^{11}$.

\section{Le comité au travail}

5 Les séances du comité se tenaient en général le soir, après celles de la Convention et se prolongeaient parfois tard dans la nuit. Les projets de loi qui y étaient rédigés ne pouvaient être présentés à la Convention avant d'avoir été discutés et arrêtés dans le comité général - soit en assemblée plénière, laquelle se réunissait les mardi et samedi de chaque semaine ${ }^{12}$. Le comité avait donc pour mission de rédiger les rapports et projets de décret que demandait la Convention nationale, mais dès lors, il préparait aussi les projets de «lois générales qui pouvaient être discutées et arrêtées sans attendre la Constitution ». Lorsque celle-ci serait acceptée, il était prévu de former de nouvelles sections pour examiner les lois existantes dans leur rapport avec les bases de la constitution.

En août 1793, une nouvelle réorganisation réduisait le nombre de membres du dit comité. Le Comité de salut public en désigna un certain nombre en septembre suivant, auxquels s'ajouta en octobre Bézard, ce qui portait à dix-sept l'ensemble des commissaires. Les bureaux comprenaient tout d'abord huit commis. En prairial an II, leur nombre passa à dix-huit ${ }^{13}$. Après le 9 thermidor, l'ensemble fut remanié par le décret du 7 fructidor an II, lequel créait seize comités, dont un Comité de législation, composé de seize membres. Il devait surveiller les administrations civiles et judiciaires, opérer le recensement et la classification des lois, et prendre les mesures d'exécution nécessaires. Le 17 fructidor suivant, le comité précisait lui-même ses attributions. Aux projets de loi et aux affaires particulières qui lui advenaient depuis plus longtemps s'ajoutent alors les arrêtés de suspension ou de destitution des fonctionnaires publics sous sa surveillance. C'est ainsi qu'en floréal et en prairial an III, c'est au Comité de législation que sont confiées les enquêtes sur les représentants en mission et les dénonciations afférentes ${ }^{14}$. Le nombre d'affaires allant croissant, le comité est contraint de se réunir chaque soir à partir de vendémiaire an III. Durant plus d'un an encore, il poursuit sans relâche ses travaux et conçoit la plupart des lois fondamentales de la Convention, au-delà même du 9 Thermidor. L'entrée dans l'ère constitutionnelle de brumaire an IV met fin à cette carrière exceptionnelle ${ }^{15}$. 


\section{Les lois pénales du printemps 1793}

7 Jusqu'au 2 juin 1793, dans le Comité de législation, Merlin et Cambacérès œuvrent donc de concert avec des hommes comme Lanjuinais, La Rivière, Louvet, Buzot, Saladin ou Vergniaud $^{16}$. C'est dire qu'en mars 1793, quand il s'agit d'élaborer les lois du tribunal criminel extraordinaire, le Comité de législation comprend des Girondins influents, qui sont coresponsables de la mise en place des premières mesures de salut public ${ }^{17}$. Depuis le 27 février en effet, des mouvements sont perceptibles à Paris, qui inquiètent la Convention. Elle a donc demandé à son comité un projet de décret pour punir et réprimer le pillage et la provocation au pillage. Le 6 mars, Garran-Coulon du dit comité propose déjà de mettre en activité les deux sections du tribunal de Paris, afin d'accélérer la marche de la justice. Entre-temps, le comité avait projeté d'exiger la peine de fer à l'encontre de ceux qui encourageaient au pillage et la peine de mort quand celui-ci aurait eu lieu. Le 9 mars suivant, suite à la séance exceptionnelle de la Convention sur la composition et l'organisation du tribunal criminel extraordinaire, Cambacérès propose qu'il soit «formé un tribunal national révolutionnaire qui ne soit établi que pour juger des peines les coupables conspirateurs et contre-révolutionnaires ; qui ne soit établi que pour assurer la révolution et qui ne soit pas censé entrer dans le plan de l'organisation judiciaire; que les juges de ce tribunal instruisent et jugent publiquement le procès des accusés sans jurés ${ }^{18}$. La discussion s'engage sur ce point délicat: $y$ aura-t-il ou non un jury ? La majorité des membres du comité pense malgré tout qu'il est nécessaire et que les jurés doivent venir de tous les départements ${ }^{19}$. En attendant leur élection, ils seront pris parmi les citoyens de Paris.

Depuis septembre 1792, le comité était aussi chargé de redéfinir la notion d'émigré. La loi devait porter contre "les véritables ennemis naturels, reconnus dans la classe des privilégiés ", à savoir les ci-devant nobles et les prêtres qui avaient plus que quiconque à pâtir du nouvel ordre. Le comité avait arrêté que c'était à la personne elle-même à prouver qu'elle n'était pas émigrée. Quant à la définition, elle était fort simple : «toute personne qui a fui la patrie par lâcheté ou trahison $»^{20}$. Le projet définitif sera arrêté bien plus tard: le 7 février 1793. Un autre problème se présente en octobre 1792: la Convention demande à son comité de penser le mode de jugement du roi, à partir de deux bases : Louis XVI peut-il être jugé21 ${ }^{21}$ Et si c'est le cas, peut-il l'être par la Convention nationale ? Le comité répond affirmativement, à la majorité absolue des voix. A partir de novembre 1792, Cambacérès s'occupe également de l'organisation du tribunal et de la procédure civile. Début janvier, c'est le sort des enfants naturels qui retient son attention, tandis que Lanjuinais s'est vu confier le projet sur la majorité civile et politique. Quelques jours plus tard, le comité doit statuer sur les visites domiciliaires et la défense générale. Ensuite, ce sont les fournisseurs aux armées qui sont l'objet des discussions. Le 23 janvier 1793, un projet de tribunal criminel du département de Paris est jugé nécessaire, en raison de la vaste démographie de la capitale. Le 27 février, on l'a dit, la Convention demande à son comité un projet de décret afin de réprimer le pillage et l'incitation au pillage. Deux projets de tribunaux extraordinaires sont alors conçus par Lesage et par Robert Lindet, tous deux membres du Comité de législation. C'est le dernier qui l'emporte dans la séance de la Convention du 10 mars. Pendant ce temps, Cambacérès peaufinait le texte et le présentait aux membres du comité. Le 9 du même mois, il plaidait à la Convention tout à la fois pour l'organisation immédiate du tribunal révolutionnaire et l'exécution rapide des lois ${ }^{22}$. C'est à lui aussi qu'est due l'introduction de la catégorie 'hors 
de la loi' du 19 mars dans la loi contre les rebelles : « Tous les rebelles pris les armes à la main seront mis à mort dans les vingt-quatre heures $»^{23}$. Une commission militaire prononcerait le jugement, sans aucune procédure criminelle et sans jury. Cambacérès ajoutait : « le projet contient des mesures sévères et il en coûte à votre comité de vous les proposer $\aleph^{24}$, mais les circonstances étant ce qu'elles étaient ${ }^{25}$, il le proposa malgré tout et la Convention accepta le projet avec beaucoup moins de répugnance qu'elle n'en avait manifesté le 10 mars précédent.

Entre-temps, deux membres du Comité de sûreté générale étaient venus consulter celui de Législation pour délibérer sur les mesures à prendre contre les étrangers résidant en France et contre les Français de séjour à Paris qui n'y avaient pas leur domicile. De même étaient poursuivis les vagabonds et les gens sans aveu ${ }^{26}$. Cette première délibération du 15 mars 1793 fut suivie le 20 par une nouvelle discussion entre les trois comités, avant que le projet ne soit porté à la Convention et arrêté le lendemain ${ }^{27}$. Trois jours plus tard une loi était proposée contre «tous les genres de prêtres et de moines qui n'auraient pas prêté serment ». Ils étaient enjoints de quitter la France et de ne pas y revenir sous peine de mort. Dans d'autres cas, ils seraient déportés à l'île Saint-Vincent ${ }^{28}$. Seuls seraient exceptés les infirmes et les vieillards. Mais le problème du jugement des contrerévolutionnaires n'était pas encore résolu. Le 5 avril, il est de nouveau à l'ordre du jour. Se pose alors la question de savoir si le tribunal extraordinaire sera ou non un tribunal d'instruction. Le Comité de législation s'accorde pour répondre par la négative.

\section{Les multiples activités du comité}

10 Depuis le 26 mars précédent, alors que la mise en activité du tribunal extraordinaire préoccupait les esprits, les membres du comité examinaient également un projet relatif à la loi qui défendait aux députés de faire des journaux. Et ils réunissaient les pièces et dénonciations concernant Marat. C'était au Comité de législation en effet qu'il revenait d'établir l'acte d'accusation contre l'Ami du Peuple. Il en fera de même pour Brissot en août suivant par l'intermédiaire de Merlin de Douai, de Guyot et de Hentz ${ }^{29}$. Le 13 avril, Delaunay a terminé le rapport relatif à Marat et conclut au bien-fondé de l'accusation, ce qui est ensuite approuvé par la Convention. L'Ami du Peuple en effet aurait provoqué au meurtre, à l'assassinat ; il aurait stimulé les violences populaires et le pillage et menacé la souveraineté du peuple en appelant de ses vœux un tribun militaire, un dictateur, un triumvir. Chaque chef d'accusation est alors successivement mis aux voix et adopté. Renvoyé au tribunal extraordinaire, Marat sera malgré tout acquitté, mais l'Assemblée nationale a commis une grave erreur en faisant arrêter un de ses membres. Elle crée un précédent, qui se retournera contre d'autres représentants. Les Montagnards se feront un devoir de le rappeler aux survivants girondins de retour après Thermidor, afin de motiver les arrestations du 2 juin et d'en minorer l'illégalitée ${ }^{30}$.

11 Parallèlement aux nombreuses lois pénales contre les émigrés, les ci-devant nobles, les prêtres réfractaires, les fabricants de faux assignats, et outre celles relatives aux peines à infliger dans le cas de tentatives de crimes non consommés, le Comité de législation traite les pétitions des particuliers et les questions qui leur parviennent des tribunaux criminels des départements au sujet de telle ou telle procédure. Il tranche aussi sur l'émission de faux assignats. Pour une contrefaçon de 25 livres, le suspect recevra une peine de 6 années de fer; de 4 années pour des assignats inférieurs à cette somme. En cas de récidive, le criminel verra ces peines doublées. Merlin de Douai se voit ainsi confiés 
plusieurs rapports sur les peines relatives " aux individus qui discréditent les assignats " dans les départements du Haut et du Bas Rhin ${ }^{31}$. Et surtout, sous la direction de Cambacérès, le comité se préoccupe du code civil. A plusieurs reprises, Cambacérès présente son rapport sur les enfants naturels, sans beaucoup de succès. Soit l'assemblée générale n'est pas au complet, soit la discussion est rapportée en raison d'autres priorités. Les premiers articles n'en seront votés qu'en brumaire an II. C'est-à-dire quelques jours seulement avant l'abandon soudain du code par la Convention, le 3 novembre $1793^{32}$.

Dès août 1793, Cambacérès avait pourtant pensé faire voter son premier projet de Code civil. L'heure hélas n'était pas à une législation pacifiste. Devant les troubles qui se répandent dans les départements et qui font craindre une guerre civile généralisée, le 16 août, la Convention demande à son comité « un rapport sur le mode d'exécution pour la mise en état d'arrestation des personnes suspectes ». Merlin de Douai est désigné pour le rédiger. Trois jours plus tard, il présente en assemblée plénière un premier rapport qui est ajourné. Deux membres lui sont adjoints pour corriger ce texte initial et « présenter de concert de nouvelles vues sur cet objet important ». Ce sera chose faite le 29. Le projet est alors adopté et présenté à la Convention ${ }^{33}$. Le 31 août, la Convention le fait encore modifier avant de l'accepter définitivement le 17 septembre suivant. Ce décret passera à la postérité sous le nom de loi des suspects. Mais c'est dire que la question préoccupait l'assemblée depuis début août, et non seulement depuis le 5 septembre. Elle avait été soulevée par les députés des assemblées primaires, de séjour à Paris, pour l'acceptation de la Constitution et la fête de l'Unité et de l'Indivisibilité de la République du 10 août. Mais c'est dire aussi que deux des lois les plus répressives et les plus révolutionnaires - ou fautil dire terroristes? - ont été conçues et défendues par des hommes qui sont longtemps passés pour des modérés, voire pour des opportunistes.

\section{De la responsabilité des légistes révolutionnaires}

Opportunisme ou conviction sincère $?^{34} \mathrm{Car}$, que ce soit Merlin ou Cambacérès, aucun des deux juristes ne reniera ces textes à la chute de Robespierre ${ }^{35}$. Bien au contraire. Merlin défendra le tribunal révolutionnaire et la justice sévère et inflexible qu'il implique jusqu'à sa suppression ${ }^{36}$. Le 23 thermidor, il se fâche même qu'on veuille modifier le dit tribunal, alors qu'il s'agirait simplement d'en supprimer les abus, dus selon lui à la loi du 22 prairial. Pourtant, le 14 thermidor précédent, Merlin fulminait qu'on veuille si rapidement supprimer cette fameuse loi, car disait-il, « en vertu de quelle loi les juges exercent-ils leurs fonctions? En vertu de la loi du 22 prairial. Or, si vous rapportez cette loi, vous n'avez plus de juges! $!{ }^{37}$. Quant à Cambacérès, en brumaire an III, il plaide encore et toujours pour le maintien de la loi des suspects : «la loi dont on parle ici fut rédigée en très grande connaissance de cause et après un examen très approfondi... ce n'est qu'en la maintenant exactement que la Convention pourra conserver à l'ordre du jour la sévérité et la justice nécessaires aux temps où nous nous trouvons $»^{38}$. Certes, lui aussi condamne les abus induits par la loi de prairial, «mais pour cela il ne faut pas détruire l'institution. Il faut les prévenir. C'est ce que fait la Convention depuis le 9 thermidor... la Convention ne doit pas permettre que la révolution rétrograde $»^{39}$. Le 23 thermidor précédent, Cambacérès soutenait son collègue Merlin et ne craignait pas à cette date de faire l'éloge du gouvernement révolutionnaire, «ce palladium de la république » : « cette salutaire conception, inconnue à tous les peuples qui avant nous ont tenté d'être libres, donna bientôt à tout une face nouvelle $»^{40}$. L'inflexibilité des légistes était également perceptible le 13 ventôse an II, quand Merlin conseillait au Comité de 
législation de faire juger les personnes accusées de vol par le tribunal révolutionnaire " pour ne pas laisser traîner les choses en longueur $~^{41}$. Et que dire de l'intervention du 25 frimaire an III, quand, au milieu des tumultes et des protestations, Merlin se refuse fermement à ce que les Girondins proclamés hors la loi soient réintégrés? «Avez-vous fermé les portes des Jacobins", s'écrie-t-il, "pour ouvrir celles du Temple $»^{42}$ ? Ces interventions suffisent-elles à donner raison à Robert Lindet? Dans sa défense de l'an III, Lindet en effet imputait à Merlin et au Comité de législation les " assassinats judiciaires " dont on accusait les anciens membres des Comités de Salut public et de Sûreté générale. A lire leurs rapports, ajoutait-il, on observera "par quels degrés rapides le tribunal révolutionnaire devint l'effroi de la France ». C'est qu'ils firent " augmenter chaque jour les attributions et l'activité redoutable de ce tribunal $»^{43}$. A remonter dans le temps, il s'avère en effet que c'est à un rapport de Merlin qu'est due la proposition du 30 frimaire an II d'assimiler la procédure criminelle dans les départements à celle du tribunal révolutionnaire, ainsi qu'il l'avait fait antérieurement pour les tribunaux militaires ${ }^{44}$. Le 12 floréal, le Comité de législation discutait une loi proposée par Merlin, qui fut portée le 19 à la Convention. Ce jour-là, après avoir entendu le rapport du Comité de Salut public et du Comité de législation, la Convention décrétait qu'en vertu de la loi du 27 germinal, les tribunaux et commissions révolutionnaires établis dans les départements par les arrêtés des représentants du peuple étaient supprimés pour ce qui touchait aux jugements des crimes contre-révolutionnaires, lesquels seraient exclusivement du ressort du tribunal révolutionnaire ${ }^{45}$. En principe, du moins, car le Comité de salut public avait le droit d'en décider autrement, ce qui explique par ailleurs son arrêté du 21 floréal relatif à la création de la commission populaire d'Orange ${ }^{46}$.

\section{Des mœurs et des principes}

14 A suivre les interventions de Merlin, l'impression domine que ce qui lui importe, c'est que les lois soient conformes aux principes. Ceux-ci peuvent être révolutionnaires, ils n'en doivent pas moins être justes, car de la «bonté des institutions » dépendrait la garantie sociale. Le 25 fructidor an II, quand il est question de libérer les patriotes et de suspendre l'exécution des jugements ayant pour cause la révolution, Merlin rétorque qu'on ne saurait dévier des principes. Pas question en effet de libérer impunément tout citoyen: «Il y a ici une confusion d'idées qui m'étonne. Il y a trois sortes d'accusation: on peut être arrêté comme suspect pour les cas prévus par la loi du 17 septembre ; comme contrerévolutionnaire ou pour des délits dont connaissent les tribunaux criminels ». Aucun des trois chefs d'inculpation ne saurait être suspendu sans causer du tort à la loi et à la sociétée ${ }^{47}$, concluait Merlin sur un ton tranchant qui n'avait rien à envier à celui de Robespierre. Et d'ajouter: "la république ne peut se sauver quand on déviera des principes ». Le 23 germinal an III, enfin, quand il reconçoit « les bases de l'ordre social et du bonheur public ", c'est à nouveau à partir des mœurs, des principes et des lois ${ }^{48}$.

Le rapport du 8 nivôse an III sur le nouveau tribunal révolutionnaire illustre fort bien ce que Merlin entendait par principes et par justice, et dévoile en filigrane ce que le légiste reprochait à la loi du 22 prairial. Tout d'abord il en critique les abstractions perverses, qui rendaient vagues et indéterminés les délits contre-révolutionnaires ${ }^{49}$. Merlin se flatte d'y substituer une définition exacte et précise. Proclamer par exemple qu'était contrerévolutionnaire, celui qui " altérait l'énergie du gouvernement révolutionnaire », est à ses yeux aberrant, tout comme «le droit dont s'était investi un seul homme de traduire qui il 
voudrait au tribunal, sans en référer à qui que ce fût $»^{50}$. Il condamne encore le fait qu'avait été supprimée la défense devant le jury, ce «droit naturel», palladium de la liberté publique. Et puis, l'amalgame des détenus lui est insupportable, de même que la dénomination de jurés patriotes: "à la faveur de cette abstraction, toutes les autres règles étaient anéanties». Notamment un renouvellement régulier du jury, composé d'hommes de toutes sortes et issus de tous les départements. Il n'est pas non plus favorable à la « conviction morale » et souhaite lui adjoindre des preuves positives, sinon la loi serait par trop arbitraire. Enfin, scandale des scandales pour un homme du droit : la loi du 22 prairial avait fait disparaitre la graduation des peines : "la mort, la mort, toujours la mort ! ». Le nouveau projet, selon lui, remet « tous les principes à leur place » ${ }^{51}$. Dès le 23 thermidor, sur la motion de Bourdon de l'Oise, la Convention avait de fait accepté de réintroduire la question intentionnelle ${ }^{52}$. Mais c'était tout ce qu'avait toléré Merlin à l'époque. Et c'est seulement le nouveau tribunal du 8 nivôse an III qui ajoute d'autres obstacles à l'arbitraire: il prévoit un renouvellement régulier des juges et des jurés, une graduation des peines, l'abolition du délai de trois jours pour les délibérations du jury, la communication des motifs de l'arrestation à l'accusé, la présence d'un conseil et les règles élémentaires de la procédure ${ }^{53}$.

16 Ces critiques relatives à la loi du 22 prairial dévoilent donc ce qu'en pensaient en leur for intérieur les juristes de la Révolution, et ce qu'ils n'avaient osé pleinement exprimer durant les séances houleuses des 22-24 prairial $^{54}$. Sous prétexte des circonstances exceptionnelles dans lesquelles se trouvait la France, tous acceptaient l'existence d'un gouvernement révolutionnaire, sévère et énergique, doté d'un tribunal extraordinaire, mais répugnaient à l'arbitraire des lois.

17 Entre-temps le Comité de législation avait cessé de tenir à jour ses registres et procèsverbaux et surtout, il mentionnait de moins en moins le nom des intervenants et des rapporteurs ${ }^{55}$. Le 17 fructidor an II, le comité avait donc été réorganisé et ses pouvoirs accrus au détriment des deux grands comités de la Convention, et, au grand dam de Cambacérès qui continuait de plaider pour la force du gouvernement et contre l'éparpillement des pouvoirs - mais qui n'en était pas moins réélu président. Quant à Merlin de Douai, il faisait désormais partie du Comité de salut public ${ }^{56}$. Une série des archives nationales contient les derniers procès-verbaux du fameux Comité de législation des 22 fructidor an II au 22 pluviôse an III $^{57}$, lesquels traitent essentiellement les pétitions de particuliers. Le 14 brumaire an III, Cambacérès disparaît de la liste des membres et le 19, c'est Bar qui devient président. Un peu plus tard, Merlin réintègre le comité et poursuit son œuvre de légiste. C'est à lui qu'est due la nouvelle loi du 18 nivôse an III contre les émigrés - loi dont il espère bien qu'elle " jettera la terreur " parmi les ennemis ${ }^{58}$. Mais il est également un des seuls à prendre la défense de Maignet contre les attaques de Rovère et consorts. A ses yeux, Maignet n'aurait fait qu'obéir à « l'infâme » comité de salut public, mené par Robespierre ${ }^{59}$.

Le plus surprenant sans doute est que lors des règlements de compte qui se succèdent entre fructidor an II et prairial an III, aucun des deux juristes ne fut incriminé ou accusé. Que ce soit Barère, Billaud-Varenne ou Collot d'Herbois, dans leurs défenses, jamais ils n'invoquent ni Merlin, ni Cambacérès. Leurs ennemis et accusateurs ne mentionnent pas non plus les membres du Comité de législation et leur rôle dans les lois qui leur avaient valu la prison, l'exil ou la perte d'un proche ${ }^{60}$. Le seul à citer Merlin et à l'impliquer dans la mise en place des lois terroristes est donc Robert Lindet, qui avait été membre du dit comité avant de passer dans celui de Salut public ${ }^{61}$. Lui-même s'était constamment 
préoccupé des subsistances et, ne serait-ce son projet de tribunal extraordinaire de mars 1793, avait peu contribué aux lois d'exception et aux arrêtés pris par le Comité de Salut public ou celui de législation. Cela n'empêchera pas son arrestation au lendemain des journées de prairial. Il est vrai qu'il avait constamment défendu ses collègues : Barère, Billaud et Collot $^{62}$ et la politique du gouvernement révolutionnaire.

\section{La commission de codification des lois ${ }^{63}$} étaient impliqués dans la plus ambitieuse des initiatives de la Convention : la codification et le recensement des lois ${ }^{64}$. C'est l'autre face de la médaille pour ainsi dire, puisqu'y était également abordé tout ce qui avait trait à la législation civile, et non seulement la législation pénale et criminelle ${ }^{65}$. L'œuvre de la commission de codification des lois apporte un éclairage nouveau sur les préoccupations des hommes de l'an II. Elle démontre l'intérêt que les Conventionnels portaient à une mise en ordre et une meilleure diffusion des lois. Curieusement, c'est le 26 germinal an II que prend forme cette initiative novatrice, sur l'intervention de Saint-Just ${ }^{66}$. Outre des mesures de police générale contre les ci-devant nobles et les étrangers, la Convention décrète alors la nomination de deux commissions chargées de rédiger un code succinct et complet des lois rendues jusqu'à ce jour et « un corps d'institutions civiles propres à conserver les mœurs et l'esprit de liberté $\aleph^{67}$. Le premier code est donc confié au Comité de législation qui coordonne l'ensemble et crée à cet effet une commission de recensement et de classification des lois. En floréal an II, quand celle-ci se met au travail, elle constate l'existence de plus de dix mille textes, provenant des trois assemblées successives. Louis Rondonneau en est le secrétaire général et travaille sous la direction de Cambacérès, Merlin de Douai et Couthon, lesquels en sont responsables. La commission confirmée le mois suivant sur la proposition de Couthon aurait dû comprendre plusieurs sections, chacune chargée d'un domaine spécifique. Vingt-huit codes étaient ainsi prévus pour classifier, uniformiser et simplifier la législation révolutionnaire et la rendre accessible au peuple français.

\section{Une codification sur des bases républicaines}

L'ensemble sera rangé par ordre de matière, et par ordre chronologique, de sorte à en faciliter la consultation. Le 11 prairial, Couthon se réjouit de pouvoir remettre d'ici peu le plan général du code complet des lois : une législation uniforme " placée sur de véritables bases républicaines ». A cette date, deux mille décrets ont déjà été réunis, émargés et en partie copiés. Le 8 prairial, le procès-verbal de la séance de la commission de classification mentionnait déjà que le but de la Convention était de donner « au peuple français une législation complète $»^{68}$. A cet effet, les lois en vigueur seront «reformées, achevées et rendues concordantes pour que les Français puissent avoir véritablement des lois ». Dans la séance du 26 prairial, la commission en arrête définitivement la division à venir. Trois parties sont ainsi prévues : l'organisation du gouvernement, qui comprendra la constitution et le code du gouvernement révolutionnaire; la deuxième développera l'action du gouvernement et la troisième ses moyens ou sa force ${ }^{69}$. Les premiers codes livrés à la rédaction seront celui du gouvernement révolutionnaire, celui de la police, de la justice criminelle et civile. Au cours du mois suivant, le travail préparatoire est assez avancé pour que Cambacérès en présente à la Convention la teneur et l'intention ${ }^{70}$. 
21 Tout d'abord, les lois doivent garantir à l'homme ses droits naturels : «c'est cette garantie qu'il est venu chercher dans les associations politiques». L'action du gouvernement en revanche est de contenir chacun dans le cercle de ses devoirs. Par organisation du gouvernement, Cambacérès précise qu'il entend la distribution des pouvoirs : «elle donne en temps de révolution le code révolutionnaire; et pour le temps qui suit la révolution, le code constitutionnel». Le but du gouvernement ainsi conçu est ni plus ni moins l'ordre et la paix de la société, de même que les « droits imprescriptibles de l'homme $»^{71}$. Une fois les pouvoirs organisés, il «faut en déterminer l'application et l'exercice ». C'est-à-dire leur action sur la société : règlement des relations extérieures, surveillance générale ordinaire et répression. A ces mesures qui pacifieront la société s'ajoutent encore celles relatives à la prospérité et au bonheur que l'homme est susceptible d'attendre d'un gouvernement libre. Viennent alors les codes particuliers : agriculture, commerce, sciences et arts, contributions, émigration, travaux et secours publics. La seconde partie du plan est donc consacrée aux actions du gouvernement en vue d'apporter « l'abondance et la félicité ». Et de conclure sur les moyens devant assurer le succès de l'entreprise : ils consisteraient dans l'instruction publique, la force publique et les finances. Ces moyens feront la force du gouvernement. L'ensemble devait être achevé le $1^{\mathrm{er}}$ thermidor.

Les archives de la commission de classification procurent d'autres informations sur les convictions des responsables. La législation y est comprise comme étant diplomatique, politique et civile. Elle se divise en lois principales et en lois réglementaires. Les premières ne doivent contenir que les principes du droit, et peut-être quelques règles de conduite $^{72}$. Ce sont celles qui constituent l'Etat, tandis que les lois réglementaires concernent le gouvernement. Les premières doivent être prévues pour être de longue durée, contrairement aux secondes qui se modifient avec les circonstances. Le rédacteur vraisemblablement Rondonneau, mais peut-être aussi Cambacérès ou Merlin - ajoutait que ces diverses législations étaient souvent contradictoires et qu'il ne fallait donc pas les confondre. La législation diplomatique en effet règle les manières d'être et les intérêts de la République avec les nations étrangères, tandis que son corollaire politique comprend toutes les lois concernant les intérêts de la République vis-à-vis des citoyens qui la composent et s'étend à l'administration, à la police, aux domaines nationaux, aux établissements, aux travaux publics etc. Inversement, la législation civile règlemente les intérêts des citoyens entre eux. Elle n'est pas moins importante, tout en étant nécessairement subordonnée à la législation politique : "l'intérêt d'un particulier doit céder devant l'intérêt général, qui est aussi le sien ", concluait le rédacteur.

\section{La mission de Rondonneau}

Le 13 floréal, les représentants du peuple constituant la commission - Merlin, Cambacérès et Couthon - choisissent donc le citoyen Rondonneau comme secrétaire général. Il aura à sa disposition un personnel nombreux, trié sur le volet : "chaque employé doit justifier de son certificat de civisme. S'il laissait échapper le plus léger trait d'incivisme, il serait destitué sur le champ ». Rondonneau a prévu d'avoir à sa disposition 30 copistes, 20 secrétaires commis, 20 garçons de bureau ; 3 chefs et un secrétaire. L'ensemble devait coûter à l'Etat quelques 50.000 livres. Rondonneau, qui a du reste publié par la suite divers recueils de lois, comptait prendre pour base de son travail plusieurs collections comprenant toutes les lois publiées jusque-là : celle des décrets de Baudoin, la 
collection dite du Louvre; et celle publiée sous la surveillance du ministre de la Justice ${ }^{73}$. L'idée était d'en établir l'ordre chronologique; de mentionner en émargement la substance de chaque loi et les dispositions qui pouvaient s'y rapporter. Chaque loi serait recopiée sur une feuille détachée avant d'être « exactement collationnée » et classée par ordre de matière. Le 20 floréal est rédigé le règlement de police des bureaux. On attend des employés qu'ils s'affairent sans répit de $8 \mathrm{~h}$ du matin à $4 \mathrm{~h}$. de l'après-midi - il leur est interdit de s'absenter «même sous prétexte de prendre leurs repas ou d'aller dans une autre division ». Le 21 messidor, un nouveau règlement spécifie que les rédacteurs rapprocheront les lois de sorte à faire connaître les interprétations, les modifications, additions ou développements. Mais ils ont également le droit d'indiquer celles qui doivent être retranchées ou de proposer « un système nouveau dans son ensemble et dans toutes les parties lorsque celui de la matière sera vicieux ou indéterminé ». Une fois par décade, ils rendront compte de leur travail ${ }^{74}$.

Ces détails démontrent bien à quel point les légistes de la Convention étaient pressés de terminer le travail, mais aussi combien ils souhaitaient donner à la France « un système complet basé sur les principes de liberté et d'égalité ». Toute loi positive devant reposer sur un principe du droit naturel ${ }^{75}$. Cambacérès réaffirme cette conviction le 4 brumaire an III, quand il s'indigne qu'on critique ou délaisse l'initiative, sous prétexte que Couthon y avait été impliqué. Et de rappeler que c'est Merlin et lui qui en assumaient la direction: « en quinze jours, nous avons fait le dépouillement de 14.400 décrets », rappelait-il, irrité ${ }^{76}$. Couthon, chargé du code du gouvernement révolutionnaire, semble-t-il, ne venait jamais à la commission. L'ouvrage sera poursuivi, mais seuls seront terminés par la Convention le Code des délits et des peines du 3 brumaire an IV, conçu par Merlin de Douai et la loi de police générale du $1^{\mathrm{er}}$ germinal an III, présenté par Sieyès au nom des trois grands comités du gouvernement ${ }^{77}$. Entre-temps, les copistes avaient pourtant bien fait avancer le travail, ainsi qu'en témoignent les minutes du gouvernement révolutionnaire ${ }^{78}$.

\section{Le code du gouvernement révolutionnaire}

Un des codes les plus importants est en effet celui du gouvernement révolutionnaire. Mais l'initiative n'en est pas moins surprenante: codifier un gouvernement, annoncé comme étant provisoire ne promettait certes pas sa suppression sur le court terme ${ }^{79}$. Il est vrai par ailleurs que cette codification allait en fixer définitivement les termes, en assurer une meilleure application et en sanctionner les abus. Depuis plusieurs mois, BillaudVarenne se plaignait du reste que les lois ne soient ni exécutées ni appliquées " exactement $»^{80}$. Les divers comités recevaient une importante correspondance où les tribunaux formulaient leur désarroi devant l'inflation incroyable des lois, leurs contradictions ou leur redondance. Le Comité de législation notamment était accablé de pétitions et de missives sur des cas particuliers que ne savaient résoudre les autorités impliquées ${ }^{81}$. Ainsi, un des tribunaux criminels de département demande l'avis du comité sur ce qu'il convient de faire d'un prêtre déporté retrouvé dans le département en question. Le comité émet son avis : renvoyer le prévenu devant le dit tribunal. Le $1^{\text {er }}$ octobre 1793, par contre, quand un tribunal s'enquiert de la procédure à suivre relativement à un individu arborant la cocarde blanche, le comité décide de renvoyer l'affaire au tribunal révolutionnaire. Parfois, il donne son avis et renvoie l'affaire aux comités de salut public et de sûreté générale, à qui il appartient de trancher ${ }^{82}$. Enfin, une 
de ses tâches non négligeables est de prononcer sur les vices de procédure - quitte à annuler le jugement de tel ou tel tribunal. L'uniformisation des lois promettait donc de faciliter l'exécution des décrets de la Convention et de mettre fin à l'arbitraire ou à l'impunité. Sous la direction de Merlin et de Cambacérès, la commission de classification allait fixer les lois relatives aux autorités constituées, à la législation révolutionnaire, à l'administration, à la force publique et à la police et sûreté générale. Et surtout supprimer ce qui était répétitif ou contradictoire ${ }^{83}$.

En introduction à ce code, un des rédacteurs avait ajouté :

«Le code révolutionnaire est la direction organisée de la foudre du peuple. Au moment de l'explosion il se sert de sa force et de son bras pour briser les trônes et renverser les tyrans. Cette victoire obtenue, il en cimente le succès par des lois pénales contre les ennemis de la liberté et par le supplice des conspirateurs ${ }^{84}$

27 Le code précise: «le gouvernement révolutionnaire a la même action que le gouvernement ordinaire. Il a en outre une action qui lui est propre». Et puis, quasi tautologiquement: «son action comme gouvernement ordinaire est réglée par les lois ordinaires; son action comme gouvernement révolutionnaire est réglée par les lois révolutionnaires $»^{85}$. La Convention y est décrite comme le centre du gouvernement et exerce exclusivement le pouvoir législatif, lequel consiste dans la confection et l'interprétation des lois. Il est donc interdit à toute autorité et à tout fonctionnaire public de faire des proclamations et de prendre des arrêtés, sous prétexte d'interpréter la loi ou d'y suppléer. Mais cela impliquait ici encore que les lois soient connues. Sur la motion de Billaud-Varenne, la Convention avait déjà accepté la création d'un Bulletin des lois, qui serait envoyé quotidiennement aux autorités constituées de chaque département. Le code révolutionnaire en prévoit la lecture publique tous les décadis. Le hasard veut du reste que le premier numéro sorte au lendemain de la loi du 22 prairial et publie cette dernière. Sa carrière n'en sera pas moins remarquable, puisqu'il se perpétuera jusqu'en 1931. Aux yeux de la Convention, ces mesures diverses devaient donc assurer le bon fonctionnement du gouvernement - qu'il soit révolutionnaire ou constitutionnel.

Le code précise également la distribution des pouvoirs et leur hiérarchie. Tout en haut de la pyramide se trouve la Convention ; juste au-dessous les comités qui sont responsables devant elle et doivent régulièrement lui rendre compte de leurs travaux. L'ensemble détermine les tâches de chacun et les autorités qui en sont responsables. A lire ces archives - qui collationnent donc des lois en vigueur - la Convention fait tout son possible pour que tout corps et tout individu doté d'un certain pouvoir rendent compte à une ou deux autorités supérieures. Le code du gouvernement révolutionnaire dévoile donc en premier lieu une volonté de contrôle et de surveillance. Il a à cœur d'éviter les abus de pouvoir, les injustices et la corruption. Les représentants en mission doivent ainsi rendre compte à la Convention de leurs opérations. Ils sont personnellement responsables des arrêtés qu'ils ont pris et sont tenus de se conformer à ceux du Comité de salut public. Il ne leur est pas non plus permis de lever des taxes révolutionnaires sans la permission de la Convention ${ }^{86}$. Qui plus est, il leur est demandé de se manifester au Comité tous les dix jours. Leurs suspensions ou remplacements de personnel sont susceptibles d'être révoqués par ce même Comité. Tous enfin, en mission ou à Paris, sont comptables à la nation de l'état de leur fortune depuis la Révolution. Les commissaires auprès des armées ont plus de liberté. Ils sont autorisés à prendre les mesures de salut public que les circonstances " peuvent nécessiter ». Leurs pouvoirs en fait sont illimités - à charge de les motiver a posteriori, surtout en cas d'échec. Les corps constitués, les agents nationaux et 
tous les fonctionnaires publics sont donc placés sous l'inspection immédiate du Comité de Salut public. Les districts, par exemple, sont tenus de rendre compte tous les six jours de l'exécution des lois révolutionnaires, notamment de leurs tâches de surveillance. Les ministres eux-mêmes sont responsables devant le Comité de salut public - notamment ceux de la Guerre et de la Marine. Ils ne peuvent promouvoir ou destituer des officiers généraux sans en avoir présenté la liste au dit comité. Quant aux agents nationaux, qui allaient remplacer les procureurs syndics de département, leur nomination est vérifiée par le district avant d'être contrôlée par la Convention. Leur tâche consiste à parcourir leur arrondissement pour vérifier que les lois sont bel et bien exécutées.

Depuis le 25 septembre 1793, le Comité de salut public revendiquait seul cette dénomination. Les autres comités dans les sections et les départements porteraient le nom de comité(s) de surveillance ${ }^{87}$. Les lois des 31 août et 17 septembre 1793 avaient permis à ces comités de surveillance de dresser la liste des gens suspects de leur section, de décerner contre eux un mandat d'arrêt et de faire apposer les scellés sur leurs papiers. Le Comité de sûreté générale est quant à lui chargé de la police générale et intérieure. Lui aussi doit rendre compte à la Convention de ses opérations. Inversement, c'est à lui que s'adressent les comités révolutionnaires, chargés de la surveillance ou de la poursuite des suspects, de l'examen des certificats de civisme, du registre des offrandes et de l'application des mesures révolutionnaires arrêtées par les deux grands comités du gouvernement. S'ils arrêtent préventivement, il leur faut inscrire les motifs sur un registre ${ }^{88}$. Les présidents des comités de surveillance et des comités révolutionnaires correspondent obligatoirement tant avec le Comité de sûreté générale qu'avec le district qui les surveille. A Paris, les comités révolutionnaires communiquent directement avec le dit comité - afin que " la police n'éprouve aucune entrave ${ }^{89}$. A la fin de chaque mois, ces comités révolutionnaires envoient l'analyse de leurs délibérations et de leur correspondance à l'organe qui est chargé de les surveiller immédiatement. Leurs président et secrétaire doivent être renouvelés tous les 15 jours et ne peuvent être réélus qu'après un mois d'intervalle. Le code précise bien qu'ils ne doivent inclure aucun noble, ni prêtre non-marié. Last but not least, le Comité de sûreté générale est le seul habilité à élargir les citoyens arrêtés.

Du sommet à la base de la pyramide, un quadrillage minutieux encadre donc le personnel révolutionnaire. S'il avait eu le temps de fonctionner effectivement avant Thermidor, sans doute les abus qu'il y a eu à déplorer auraient-ils progressivement régressé. Mais c'est dire malgré tout que la Convention était consciente de la nécessité d'un contrôle sévère des nombreuses autorités et fonctionnaires qui devaient mettre en œuvre le système ${ }^{90}$. De sa part, rien n'est laissé au hasard - sur papier du moins ${ }^{91}$. Aux tâches imparties aux diverses autorités s'ajoutent du reste les peines que celles-ci encourent en cas de négligence ou d'inexécution des lois : selon les cas, une suspension du droit de citoyen pendant trois ou quatre ans et une amende du quart ou du tiers des revenus. Mais c'est dire aussi l'immensité des tâches imparties aux deux ou trois grands comités du gouvernement, qui doivent contrôler l'ensemble des corps constitués, les divers comités populaires et les représentants en mission, sans oublier la correspondance incroyable qu'implique ce contrôle. Tâches gigantesques dont on peut se demander si elles ont toutes été assumées pleinement et qui expliquent sans nul doute les « bavures » d'un régime qui est entré dans la postérité comme terroriste.

31 Le gouvernement révolutionnaire se ressentit du 9 Thermidor, sans qu'il ait été pour autant jugé nécessaire de le supprimer. Bien au contraire, puisque, dès le 17 thermidor, le 
Comité de législation faisait parvenir un projet précédé d'une déclaration des principes du gouvernement révolutionnaire, "pour servir de garantie aux droits et à la liberté du citoyen». Projet apprécié du "bon docteur» Lanthenas qui se félicite des mesures nouvelles. Le projet en question spécifie que le gouvernement révolutionnaire consiste dans «la centralisation de tous les moyens de défense de la République contre ses ennemis extérieurs, et de tous ceux de vigilance et de force contre ses ennemis du dedans", de même que dans "l'organisation prompte de tout ce qui peut sauver et renforcer la république». Les rédacteurs précisent bien que ce n'est point là une dictature, mais l'emploi de toutes les forces, le dévouement de tous les citoyens et de tous les bras au salut de la patrie - ce qui suggère que sont réquisitionnés l'ensemble des Français, ce qui est vrai, on va le voir. La forme donnée au gouvernement révolutionnaire est ensuite motivée par les circonstances périlleuses auxquelles la France est exposée. Au centre de ce gouvernement se trouve une fois encore la représentation nationale : «le centre de l'opinion publique, de la morale et de l'instruction républicaine ». Quiconque tentera de l'avilir, sera "réprimé avec [...] vigilance et puni avec [...] sévérité »" L'expérience récente permettrait, selon les auteurs, de corriger ce qui y était défectueux, de rendre à la représentation nationale l'unité de sentiments et d'action que les factions lui avaient ravie. Un des principes fondamentaux stipule que «tout moyen était bon quand on y coopérait de bonne foi ", puisque c'était pour établir la liberté. Enfin, les institutions républicaines contribueraient à rétablir l'union et à préparer la paix et la prospérité. Ces institutions - morale républicaine et instruction publique - sont décrites alors comme les moyens du gouvernement révolutionnaire. De fait, plutôt que de formuler des principes, les douze articles de la déclaration rédigés par le Comité de législation cherchaient à perpétuer le gouvernement révolutionnaire, tout en le dissociant de ses antécédents "terroristes". La nuance était subtile et le texte guère convaincant, ce qui n'était sans doute pas son objectif premier.

\section{Contrôle et surveillance}

La justice participe évidemment du gouvernement révolutionnaire. Le code prévu s'en souciait donc au plus haut point et remettait de l'ordre dans toutes les lois décrétées jusque-là. Notamment les grandes mesures pénales de la Convention de mars et d'avril 1793, quand avaient été créé le tribunal criminel extraordinaire et proclamée la peine de mort pour crime contre-révolutionnaire ${ }^{93}$. La section des peines reprend donc ces lois antérieures, les réunit et les ordonne. Les copistes et les secrétaires semblent du reste avoir eu du mal à concevoir un ordre satisfaisant. Le plus souvent, les minutes du code réunissent côte à côte des décrets qui ne sont ni forcément complémentaires, ni forcément compatibles. Ainsi au décret sur le jugement du tribunal révolutionnaire sans recours au Tribunal de cassation succède un article sur les autorités constituées qui sont toutes soumises à l'inspection immédiate du Comité de salut public, tandis que les personnes privées le sont au Comité de sûreté générale. Autre exemple de lois cette fois quelque peu contradictoires. A l'article qui contraint les femmes à arborer la cocarde tricolore succède un article qui proscrit toute tentative pour obliger « les citoyens de se vêtir d'une manière particulière $\aleph^{94}$. Personne ne parut s'apercevoir de la contradiction.

L'essentiel des articles quoi qu'il en soit concerne ici encore la surveillance et le contrôle des autorités. Ainsi un article sanctionne-t-il les abus d'autorité et la prévarication des fonctionnaires par cinq ans de fer et des confiscations ou amendes. Un autre leur interdit 
de faire des proclamations, de prendre des arrêtés ou d'interpréter ceux qu'ils reçoivent. Une section spéciale concerne l'agriculture et les précautions à prendre pour que le peuple ne manque pas de pain. Les grains eux-mêmes sont surveillés et recensés. Le 4 mai 1793, une première loi sur le maximum a déjà été décrétée, tandis qu'en août suivant, la Convention publie une liste des produits de première nécessité qui ne doivent pas quitter le territoire de la République. De même les ouvrages d'orfèvrerie et de joaillerie en or et en argent sont interdits d'exportation ${ }^{95}$. Parmi les autres sections du code se retrouvent les finances et les fonds publics. Une fois encore, la Convention a arrêté qu'aucune taxe, aucun emprunt forcé ou volontaire ne pourrait être levé sans un décret approuvé par elle. Il est de même interdit aux autorités de disposer des fonds publics ou d'en changer la destination sans autorisation. Quant aux taxes, elles sont progressives et peuvent s'élever à plus de $50 \%$ quand les revenus sont élevés. Ainsi pour un revenu de 11.000 livres, elles se montent à 6500, alors qu'un revenu de 1500 livres n'en rapporte que 100 à l'Etat.

Parmi les mesures révolutionnaires recensées par Rondonneau, celles relatives à la réquisition apparaissent comme extrêmement importantes. La France tout entière en effet est appelée à participer ${ }^{96}$ : les jeunes citoyens ensemencent les terres et s'occupent de la mouture des grains; les imprimeurs et les ouvriers fabriquent ou réparent les armes; les hommes mariés forgent les canons ; les femmes font les tentes et les habits, et servent dans les hôpitaux; les enfants mettent le linge en charpie; les cordonniers consacrent leur temps à faire des souliers pour les soldats; les savants inventent des moyens pour améliorer l'armement et les balles; les artistes enfin sont réquisitionnés, tout comme la gendarmerie, chargée de traquer les déserteurs. Bref, « jusqu'au moment où les ennemis auront été chassés du territoire de la République française, tous les Français, conformément à la loi du 23 août 1793 restent en réquisition permanente ${ }^{97}$.

Pour compenser la suppression des armées révolutionnaires arrêtée le 14 frimaire an II, un décret stipule également la formation d'une garde dans chaque grande ville choisie parmi les citoyens les moins fortunés, lesquels seront armés et salariés aux frais de la République. La section " des suspects » n'est pas moins intéressante. Elle comprend six catégories $^{98}$. Les comités de surveillance sont censés les rechercher et les dénoncer aux commandants de la force publique qui les arrêtent. Néanmoins, et cela prouve une fois de plus la prudence de la Convention, pour décider de l'arrestation d'une personne, les membres du comité en question doivent être sept au minimum et ne prendre une décision qu'à la majorité absolue des voix. Les comités de surveillance sont sommés du reste d'envoyer au Comité de sûreté générale l'état des personnes qu'ils auront fait arrêter avec les motifs de l'arrestation et les papiers qu'ils auront saisis. Qui plus est, " ceux qui auraient agi par rancœur ou vengeance » seront dénoncés au Comité de sûreté générale et punis en conséquence.

Le décret du 23 ventôse avait également prévu la création de six commissions populaires " pour juger promptement les ennemis de la Révolution détenus dans les prisons ${ }^{99}$. Deux sont créées en floréal. Les autres ne verront pas le jour, puisque la loi n'est réactualisée que les 4 et 6 thermidor. Quelques jours plus tard, l'institution elle-même sera condamnée, car associée à Saint-Just qui en avait pris l'initiative. Elle vaut cependant la peine d'être notée, car elle confiait aux simples citoyens une partie non négligeable de la police. Parallèlement aux comités révolutionnaires et aux comités de surveillance qui étaient l'œil de la vigilance, les deux commissions populaires dites du Museum poursuivaient les suspects et examinaient qui était ou non passible d'arrestation. Une fois cela découvert, elles envoyaient les détenus au tribunal révolutionnaire et faisaient 
parvenir leurs décisions aux Comités de salut public et de sûreté générale qui y apposaient leur visa. C'est dire que les deux comités qui avaient présidé à leur création avaient pris des précautions afin que les suspects soient jugés promptement, mais non précipitamment. Une troisième disposition leur prescrivait de présenter les tableaux des patriotes arrêtés ${ }^{100}$, pour qu'on pût en ordonner la mise en liberté définitive. Les commissions populaires jugeaient non seulement les détenus de Paris, mais aussi ceux de tous les départements, ainsi que le stipulait la loi du 27 germinal ${ }^{101}$. Leurs activités étaient si intenses qu'au lendemain de Thermidor, elles seront soupçonnées d'arbitraire et supprimées en conséquence.

Une troisième commission populaire avait été établie à Orange pour accélérer les jugements des suspects du Vaucluse. Mise en activité le 15 prairial sur un arrêté du Comité de salut public du 21 floréal ${ }^{102}$, elle s'avéra en vérité peu respectueuse des lois votées à Paris. Le 12 messidor, en effet, un agent de la Convention écrivait pour s'en plaindre, que sur douze suspects, neuf seulement étaient condamnés à la peine capitale, deux ayant été déportés et le dernier mis en détention. L'agent, Agricola Nouveau, dénonçait ce fait comme contraire à la loi du 22 prairial, laquelle ne connaissait plus que la peine de mort ${ }^{103}$.

La législation révolutionnaire était donc en partie entre les mains de simples citoyens auxquels étaient confiées la surveillance, la recherche et la poursuite. C'était impliquer le peuple dans la révolution et lui confier de vraies responsabilités, mais c'était aussi courir le risque de trop d'indulgence, ou inversement, de trop grande sévérité. C'était sans doute encourager les vengeances personnelles et favoriser l'assouvissement d'intérêts privés. Les comités de la Convention en étaient conscients, mais avaient-ils réellement les moyens d'y remédier? Les lois tendaient certes à y pallier. Encore devaient-elles être fidèlement exécutées et surtout bien diffusées. Les abus constatés - pour le meilleur et pour le pire ${ }^{104}$ - semblent bien prouver que le système était loin d'être parfait.

\section{Une société juste et équitable}

Outre le code révolutionnaire, la commission de classification des lois conçoit donc des codes divers, touchant aux diverses facettes de la société. Celui des secours publics est assez avancé en floréal, quand Barère en fait un long rapport à la tribune ${ }^{105}$. Il est ainsi prévu de donner des secours aux familles des défenseurs de la patrie, aux citoyens acquittés par le Tribunal révolutionnaire, mais aussi aux orphelins, infirmes, vieillards et démunis ${ }^{106}$. Pour les recenser, des commissaires de la Convention ont alors pour tâche de faire accélérer la confection des rôles exigés par la loi du 4 mai 1793 pour ces secours qui s'élevaient de 25 à 60 livres par an ${ }^{107}$. Le 8 messidor suivant, il est même question de la formation d'un livre de bienfaisance nationale qui comprendrait non seulement des secours pécuniaires, mais aussi des secours à domicile. Ce genre de lois fait partie de ce que les contemporains appelaient les lois démocratiques. Elles suggèrent une volonté d'améliorer le sort des pauvres et de récompenser les patriotes pour leurs sacrifices, et, dans le même temps, elles visent à souder les intérêts populaires à la Révolution ${ }^{108}$.

Quant aux autres codes, ils n'ont pas été achevés en l'an $\mathrm{II}^{109}$. Pour la plupart, les matériaux sont restreints et les dossiers se limitent souvent à l'organisation générale. C'est ainsi que le code de l'émigration contient 13 pièces, concernant les travaux du bureau des dettes des émigrés du département de la Seine, et des lettres et des documents relatifs à la dite liquidation ${ }^{110}$. Celui des travaux publics comprend un rapport sur 
l'organisation des bâtiments publics, le plan général d'un code, de même que des décrets et plusieurs titres, analyses et commentaires sur le sujet. Le code militaire se compose des listes et analyses des décrets sur la cavalerie, les chasseurs, les dragons et les hussards. Il inclut les rapports et les projets sur la législation militaire, les conseils de discipline et la police. Y sont adjoints les tableaux des mouvements des armées. Y figure également tout ce qui concerne «les gendarmeries nationales" à partir de leur organisation de 1790-1791 ${ }^{111}$. Dans un dossier des archives du Comité de salut public sont également mentionnés les chapitres de plusieurs projets ${ }^{112}$ : le code des administrations civiles confié au citoyen Duvallon comprendrait les corps administratifs, les municipalités, la division, les biens communaux et les élections. Le code des sciences exactes engloberait les inventions et recherches scientifiques, les arts mécaniques, les usines, les filatures et les manufactures. C'est que, disait Barère à la tribune un beau jour de septembre 1793, «Paris a perdu une population d'aristocrates. Il faut le peupler de savants ${ }^{113}$. Le commerce n'était pas non plus négligé : le code, qui y était consacré, mentionnerait ainsi les lois sur la liberté intérieure, les exceptions en matière d'exportations, le maximum et les lois contre les accapareurs ou les délits en la matière. Le code des travaux publics, qui revenait à Rondelet, inclurait les édifices nationaux, les lois sur les monuments publics et les peines appliquées à quiconque dégraderait les monuments des arts. Le plus exhaustif se trouvait être le code criminel, attribué au citoyen Cordier, qui se composerait de dixhuit chapitres ${ }^{114}$.

41 Les travaux étaient donc vastes et fastidieux, ce qui explique leur achèvement sur le long terme seulement. Plusieurs des codes seront en effet poursuivis soit par le Directoire, soit par Napoléon, qui, sur ce point également, était bien l'homme de son siècle, puisque lui aussi désirait codifier l'ensemble des lois, qu'elles soient civiles ou criminelles, commerciales, militaires ou judiciaires ${ }^{115}$. Ici encore, c'est Louis Rondonneau qui en assure la publication - et peut-être même la rédaction. Mais cela prouve avant tout l'intérêt et le sérieux que manifestait la Convention relativement à la mise en ordre des lois, nécessaires à la reconstruction de la France, alors même qu'elle affrontait les puissances de l'Europe.

\section{Les lois démocratiques}

Parallèlement aux codes, un document anonyme des archives du Comité de salut public recense les lois démocratiques votées par la Convention ${ }^{116}$. Celles-ci constituent en somme le troisième volet du triptyque - puisque triptyque il semble bien y avoir. Elles sont autant de preuves du souci pressant des représentants de pourvoir aux besoins du peuple et à sa sécurité. Les dates mentionnées témoignent bien que l'époque montagnarde tenait à en revendiquer la paternité. La première loi recensée date en effet du 4 juin 1793. C'est celle qui accorde des pensions alimentaires et des secours aux veuves de militaires morts aux combats ou par suite de blessures. Suivent en juin le décret qui détermine le mode de partage des biens nationaux ; celui qui abolit la loi martiale ; celui qui fixe le montant ${ }^{117}$ des récompenses nationales données aux défenseurs de la patrie et qui organise les secours à fournir annuellement aux enfants et aux vieillards; qui accorde des pensions de retraite aux militaires blessés dans les combats et qui établit dans chaque département une maison de secours destinée à recevoir les pauvres, les infirmes ou les aveugles des deux sexes. En juillet, est décrétée la suppression sans indemnité de toutes les redevances ci-devant seigneuriales, des droits féodaux censuels et de ceux qui avaient été conservés par le décret du 28 août 1792 . Le 14 août suivant, il est arrêté des 
indemnités pour les citoyens qui auraient éprouvé des pertes suite à l'invasion des ennemis et le 19 août des indemnités pour ceux qui seraient chargés d'enfants abandonnés.

Le mois de septembre est plus favorable encore aux démunis. Le 9, la Convention accorde quarante sous aux citoyens indigents pour assister aux assemblées de leur section; le 10, elle ordonne que les citoyens dans un besoin pressant de grains puissent s'en faire prêter par les greniers de la république de leur arrondissement; le 11, c'est le décret sur le maximum qui sera fixé pour les objets de première nécessité le 29 septembre suivant. Le ministre de l'Intérieur recevrait trois millions pour faire diminuer l'effet des accaparements et le prix des denrées. Le 13, une loi est votée permettant d'acquérir « des biens d'émigrés à concurrence de 500 livres en faveur de ceux qui n'ont point eu partage des biens communaux ", les dits biens payables en vingt ans sans intérêt. Les acquéreurs de biens nationaux obtiendraient une prime s'ils se libéraient avant échéance. Le 16 septembre, une autre loi chargeait les municipalités de faire cultiver les terres des défenseurs de la patrie. Le 19, un décret accordait des secours aux patriotes des Tuileries et environs pour les pertes éprouvées lors du 10 août.

En octobre, était réglée l'interprétation de l'article du 10 juin sur le mode de partage des biens communaux. Les acquéreurs de biens de la liste civile étaient autorisés à payer moitié en inscription sur le grand livre et moitié en assignats le prix de leur acquisition. Un décret portait sur l'extinction de la mendicité; un autre stipulait que le défaut de paiement des amendes prononcées par la police correctionnelle entraînerait une détention des personnes insolvables qui ne dépasserait pas un mois. Durant ce même mois, la pêche du maquereau et du hareng était permise à tout Français, tandis que des écoles allaient être ouvertes dans "toute la république à raison de la population ». Des articles additionnels de brumaire an II complétaient cette loi favorable à l'instruction publique. Ce même mois était déclaré nul tout jugement sur les procès intentés relativement aux droits féodaux ou censuels, fixes et casuels, abolis sans indemnité et rendus postérieurement à la loi du 28 août 1792. De nouvelles indemnités étaient décrétées pour venir au secours de ceux qui avaient souffert des intempéries, des incendies ou autres accidents, de même que les familles qui avaient pâti des massacres du Champ de Mars, tandis qu'étaient adoptés plusieurs articles du code civil, relatif aux enfants nés hors du mariage.

Durant le mois de frimaire se décèle un même souci de porter secours aux démunis et de démanteler tout ce qui reste de la féodalité. S'y ajoute l'abolition de toutes les procédures instruites et de tout jugement rendu sur des faits relatifs aux insurrections populaires concernant le prix des denrées, sans oublier le nouveau décret du 29 frimaire sur l'organisation de l'instruction publique. Le mois de nivôse suivant augmente d'un tiers les secours et indemnités réservés aux défenseurs de la patrie et à leur famille et confirme l'exécution de la loi sur les secours publics. Une autre précise que les acquéreurs de biens nationaux auront dix ans pour achever le paiement en remboursant le dixième chaque année. La Convention demande également l'accélération de l'exécution de la loi pour que les terres des défenseurs de la patrie soient cultivées, et exige par ailleurs que toutes les communes de France plantent des pommes de terre. Le 28 nivôse est arrêté un décret sur les difficultés rencontrées dans l'application des lois sur les ci-devant droits féodaux. Au mois de ventôse sont votées les fameuses lois qui ordonnent le séquestre des biens des personnes détenues et reconnues ennemies de la Révolution et l'établissement par les communes d'un état des patriotes indigents auxquels sera donnée une propriété « sur les 
lieux des ennemis de la Révolution ». Aux citoyens blessés en défendant la patrie serait également accordée " une portion de terre assez considérable pour élever leur famille ». Parallèlement étaient fixés les salaires dus aux instituteurs et aux institutrices des petites écoles. Les 14 et 16 ventôse, enfin, une nouvelle somme de vingt millions était mise à la disposition du ministre pour être répartie entre les citoyens ayant éprouvé des pertes par l'invasion et le ravage des ennemis et cinq cent mille livres leur étaient ajoutées pour venir au secours des citoyens infirmes et incapables de travailler. La liste des lois démocratiques s'achève au 16 ventôse, quatre mois et demi avant Thermidor ${ }^{118}$ et ne comprend pas donc l'ensemble des lois votées par la Convention, notamment celle du 22 floréal sur les moyens d'extirper la mendicité et les secours à accorder aux indigents ${ }^{119}$ ou celle du 19 prairial qui accorde des secours aux patriotes étrangers, réfugiés en France ${ }^{120}$. Le 13 prairial, la Convention désirait déjà accélérer la distribution des secours publics, dans le même temps où elle décrétait la création de l'Ecole de Mars. Le 16, elle s'intéressait à l'uniformité de la langue française et décidait de faire rédiger une nouvelle grammaire et un vocabulaire nouveau. Tout comme les codes entrepris depuis germinal an II, cette liste - non exhaustive - témoigne avant tout des préoccupations multiples de la Convention qui ne se limitaient donc nullement à la répression ou à la surveillance. Des institutions diverses et variées faisaient leur entrée dans le paysage de la France révolutionnaire, dont allaient hériter le Directoire et le Consulat, qui orienteraient cependant la législation sur une voie moins humanitaire.

D'une part, le gouvernement était donc révolutionnaire jusqu'à la paix, et il exigeait une activité de tous les instants et de tous les Français, doublé de la poursuite et de la punition des ennemis de la Révolution; d'autre part, il y avait des récompenses, des indemnités et des pensions ; des prix équitables pour les objets de première nécessité ; des promesses de prospérité pour les démunis; mais encore des écoles, des monuments, des fêtes, des inventions, des concours, et des lois en faveur de l'agriculture et du commerce ${ }^{121}$. La codification entreprise préparait bel et bien la rentrée de la France dans l'ère républicaine. Ce n'était pas là une vague utopie robespierriste, mais des mesures pensées et mises en œuvre par les légistes des divers comités et commissions, et même si la législation n'était pas toujours fidèlement appliquée par les autorités départementales ${ }^{122}$. A voir cette boulimie d'activités, force est de donner une fois encore raison à Barère, qui regrettait la mauvaise réputation de la Convention, dont «tous les travaux ont été méconnus, toutes les intentions calomniées, tous les bienfaits empoisonnés et presque tous les résultats anéantis $»^{123}$. Et il est vrai que l'œuvre positive et constructive de la Convention a été éclipsée dans les mémoires par celle que, depuis Thermidor an II, il a été convenu d'appeler la Terreur. De cela, les Thermidoriens sont pour une grande part responsables ${ }^{124}$.

S'intéresser aux divers comités et commissions permet de mieux comprendre l'incroyable complexité du système mis en place par la Convention ${ }^{125}$ et partant, la Révolution ellemême. Il s'avère ainsi que les lois civiles et pénales étaient conçues par des juristes éminents, tels que Cambacérès et Merlin de Douai, qui fulminaient du reste quand SaintJust et Robespierre s'accaparaient ces prérogatives, que ce soit lors du décret du 23 ventôse, de l'arrêté du 21 floréal ou de la loi du 22 prairial. Cette approche rend donc leurs pleines responsabilités à des personnages jugés secondaires, qui n'en pesaient pas moins sur les décisions et sur le cours de la Révolution - laquelle ne s'est identifiée à Robespierre qu'un temps seulement ${ }^{126}$. Elle témoigne encore de ce que la législation n'était ni bâclée ni confinée entre les mains des «idéologues » ${ }^{127}$, si l'on me permet 
l'anachronisme, mais qu'elle était amplement discutée et étudiée avant d'être définitivement proclamée ${ }^{128}$. La fameuse loi des suspects du 17 septembre 1793, inspirée du projet de Merlin du 19 août précédent en est un exemple éloquent, tout comme celle du Tribunal révolutionnaire qui donna matière à bien des débats et à plusieurs projets. Un autre exemple pourrait être la rédaction de l'acte d'accusation contre Brissot, rédigé par trois membres du Comité de sûreté générale, assistés de trois membres du Comité de législation - Merlin, Hentz, Guyot ${ }^{129}$. La correspondance du Comité de législation démontre encore que bien des autorités départementales cherchaient sincèrement à suivre les ordres du gouvernement et qu'elles s'adressaient souvent en haut lieu afin de ne pas faire d'erreurs. Elle démontre encore que les cas particuliers étaient traités avec soin et les décisions jamais prises à la légère ${ }^{130}$. Last but not least, l'examen des textes décrétés par la Convention et conçus par le Comité de législation et la commission de classification des lois dévoile que législations civile et pénale marchaient de concert et que l'une et l'autre étaient inséparables ${ }^{131}$. La codification entreprise depuis germinal an II préparait l'entrée en république démocratique de la France. Et cette législation portait non point sur la répression en tant que telle, mais avant tout sur la surveillance et le contrôle des citoyens et des autorités. Parallèlement, elle construisait une société juste et équitable, qui devait récompenser les patriotes des sacrifices faits à la patrie ${ }^{132}$. De ce point de vue, il est difficile d'y percevoir une terreur véritable, à moins d'interpréter les lois coercitives exigées par les circonstances comme telles ${ }^{133}$. Des lois fondées sur les droits de l'homme, et, rappelons-le, conçues et décrétées pour la plupart par des légistes, connus et reconnus, et non seulement par Couthon, Saint-Just et Robespierre ${ }^{134}$.

\section{NOTES}

1. Seuls les historiens du droit abordent cette facette de la Révolution, mais fondent le plus souvent leur interprétation globale sur celle des historiens de la Révolution. Notamment sur les ouvrages dits révisionnistes, qui demandent pourtant à être revus et corrigés, parce qu'ils méconnaissent le contexte, les précédents français ou étrangers, et la rhétorique propre à l'époque. Sur les dangers à méconnaître la réalité beaucoup plus complexe, où s'entremêlent stratégies rhétoriques, contraintes discursives et visées idéologiques, voir P. CAMPBELL (ed.), The Origins of the French Revolution, Palgrave, 2006, p. 7-9. Notamment l'article de Marisa LINTON, «The Intellectual Origins of the French Revolution », p. 139-159.

2. Il ajoutait «Vous avez compris l'œuvre politique de la Convention, dont le malheur fut de n'être pas entendu et de parler une langue non comprise, ce qui la força à prendre des mesures rigoureuses ». Mss. No.144. Bibliothèque historique de la Ville de Paris. Léonard Gallois écrivait alors une Histoire de la Convention par elle-même en 8 volumes (Paris, 1836-1848). Pour une première tentative de réhabilitation du gouvernement conventionnel, voir le Journal des Hommes libres, Messidor an VII, no.8, p. 32.

3. Etrangement donc, la législation révolutionnaire a été très peu étudiée. Françoise Brunel pourtant insistait bien sur cette facette de la Révolution, sans s'intéresser du reste au Comité de législation ou à la codification et sans focaliser sur les détails. Voir Thermidor. La chute de Robespierre, Bruxelles, 1989, p. 45 et p. 61-64. Inversement, Carla Hesse a bel et bien consacré des 
études aux lois révolutionnaires et à leur prolifération, mais pour se concentrer sur les lois pénales et la série $\mathrm{W}$ des Archives nationales, ce qui évidemment l'incite à conclure sur le caractère éminemment répressif de la législation révolutionnaire. « La logique culturelle de la loi révolutionnaire ", Annales HSS, vol. 4, 2002, p. 915-933. Les tables ou répertoires publiés par les contemporains suggèrent tout autre chose et nous y revenons plus loin. Voir la Table générale alphabétique et raisonnée des matières contenues dans le répertoire de jurisprudence et dans le recueil alphabétique des questions de droit de M. Merlin par L. Rondonneau, Paris, 1829 ou Répertoire des lois et des arrêtés du gouvernement de 1789 à l'an XI par ordre alphabétique, chronologique et par classement de matières de Guillaume Beaulac, Paris, 1804.

4. Dans un article de fond, Georges Bourgin s'en plaignait déjà dans "Le Comité de législation », Nouvelle Revue historique du droit français, t. 35, 1911, p. 624-648.

5. Pierre lascounes et ALII, Au nom de l'ordre. Une histoire politique du code pénal, Paris, 1989, p. 97-102. Voir aussi Archives parlementaires, $1^{\mathrm{e}}$ série, t. 8, p. 641. Ce premier comité comprenait de Beaumetz, Fréteau, Tronchet, Le Berthon, Thouret, Target et Lally-Tolendal.

6. Un chapitre succinct est consacré au Comité de législation dans Jean-Louis HALPÉRIN, L'impossible code civil, Paris, 1992, p. 115-122. Voir aussi Philippe SAGNAC, La législation civile de la Révolution française. Essai d'histoire sociale, Paris, 1954.

7. La première se concentre sur les conventions matrimoniales, l'administration des biens des époux, les droits respectifs des époux, les tutelles, les curatelles, et sur l'autorité paternelle. La deuxième présentera des lois sur les successions directes, sur les enfants nés hors du mariage, l'adoption et les successions collatérales. La troisième se consacre aux donations, testaments, substitutions, moyens d'acquérir et de conserver. Quant à la quatrième, elle est vouée aux conventions, obligations, hypothèques, rentes, restes de la féodalité, et aux changements à faire au code pénal. AN- D III 380.

8. Archives parlementaires, $1^{\mathrm{e}}$ série, éd. J. Madival \& E. Laurent, Paris, 1867, t. 66, p. 4.

9. Sur Merlin de Douai, voir Hervé Leuwers, Un juriste en politique. Merlin de Douai, Arras, 1996, p. 75.

10. Sur ces détails, A. DEJACE, Des règles de dévolution successorale sous la Révolution, E. Bruylast, LGDJ, 1957, p. 280-282. Informations que je dois à Anne Simonin, que j'ai plusieurs fois consultée et que je remercie en conséquence. Elle aussi s'intéresse à la législation. Voir le dossier « Regards croisés : Si l'on parlait de république ", $A H R F, n^{\circ} 364,2011$, p. 211-238. En particulier, p. 219.

11. AP, t. 89, p. 169.

12. AN D III-380.

13. Georges BOURGIN, op. cit., p. 634. En prairial an II, il y avait trois sections avec dans chacune un chef, un sous-chef et trois commis.

14. Un nombre important de cartons d'archives est consacré à ces dénonciations et aux dossiers des représentants en mission, de même qu'aux observations des membres du comité. Ces poursuites s'amorcent donc après la réinsertion des Girondins dans la Convention et les journées de Germinal et de Prairial. AN-D III 343-358.

15. G. BOURGIN, p. 640-641.

16. Aux côtés de Berlier, Garran-Coulon, Bézard, Génissieux, Lacoste, Oudot, Florent Guyot, Charlier, Azéma et Bar.

17. De même, la création du Comité de salut public du 6 avril 1793 se fait d'après le rapport d'Isnard, membre du Comité de défense générale. Voir AN- AF II * 45. Procès verbaux du Comité de Défense générale.

18. Les procès-verbaux des séances sont consignés dans la série AN D III* 54-58 - non paginée.

19. Ces discussions ont donc lieu avant que le débat ne se déplace vers la Convention. Or, dans le Comité de législation, les décisions sont souvent peu contestées. Les procès-verbaux en tout cas ne reproduisent pas les dissensions - contrairement à ceux de la Convention. La question du jury 
sera également discutée à la Convention dans la séance du 10 mars, mais ce qui frappe ici, c'est que, dès cette date, Cambacérès proposait qu'il n'y en ait point.

20. AN D III-380.

21. AN D III* 54.

22. Voir les discussions les 9 et 10 mars dans $A P$, t. 60, p.59-71. Les deux séances furent houleuses. La seconde dura 8 heures d'affilée; reprit le soir à 7 h.45 et se termina à 4 heures et demi du matin.

23. La loi était ainsi libellée : «Ceux qui auront pris part à des révoltes ou émeutes contrerévolutionnaires ... ceux qui auront pris ou prendront la cocarde blanche ou tout autre signe de rébellion sont hors de la loi. En conséquence, ils ne peuvent profiter des dispositions des lois concernant la procédure criminelle ou l'institution des jurés ". Procès-verbaux de la Convention, Paris, 1793, vol. 8, p. 88-89. Sur cette catégorie, voir la thèse d'Eric de Mari, que nous n'avons pu consulter - malgré notre demande expresse à l'auteur, dont nous n'avons pas eu de réponse. Rappelons toutefois que cette catégorie pénale existait déjà en Angleterre sous le nom de «outlaw ».

24. Archives parlementaires, t. 60, p. 331 (décret du 19 mars 1793). Voir aussi les discussions du 10 mars 1793, où plusieurs membres du Comité de législation qui ont accepté le projet lors de la discussion du 9 au comité le condamnent devant la Convention. Ainsi Lanjuinais et Buzot, tandis que Cambacérès défend vigoureusement ce qu'il appelle dès lors le tribunal révolutionnaire. Sur cette séance, note 22 .

25. Et témoignage éloquent des acteurs eux-mêmes sur les motivations qui les poussaient, il ajoutait: "Vous n'oublierez pas que les circonstances commandent presque toujours les décisions ", AP, t. 60, p. 331.

26. Une loi du $1^{\text {er }}$ août 1793 stipulait que les étrangers devaient être signalés et arrêtés, s'ils n'étaient pas domiciliés en France avant le 14 juillet 1789. Mais notons bien que le décret du 21 mars stipulait déjà l'établissement dans chaque commune d'un comité chargé de recevoir les déclarations des étrangers qui y résidaient ou y arrivaient. AN D XXXIX, 10. La méfiance envers les étrangers, facilement assimilés aux émigrés ou contre-révolutionnaires ne doit pourtant pas faire oublier que les patriotes étrangers réfugiés en France furent constamment protégés et secourus. Les Américains de séjour dans la capitale ne furent même pas inquiétés. Qui plus est, le 21 floréal an IV, la loi dite xénophobe et terroriste d'août 1793 était réintroduite, mais cette fois, en raison du « complot babouviste ». Bulletin des Lois, an IV, $\mathrm{n}^{\circ}$ 45, p. 395.

27. AN- D III* 54. La méfiance à l'égard des étrangers ne date donc pas d'août 1793. Au printemps 1793, la Convention s'inquiète de leur afflux, notamment à Paris.

28. La déportation à l'île Saint-Vincent fut proposée le 26 mars par Charlier pour les prêtres qui, ayant prêté serment, n'auraient pas de certificat de civisme. AN D III* 54.

29. AN-D III* 55. En date du 20 août 1793.

30. Journal des hommes libres, $\mathrm{n}^{\circ}$ 190, 10 germinal an III, p. 784. Voir aussi no.187, p. 769, où Leblanc rappelle les propos d'Isnard «qui voulait la ruine de Paris » ou Lecointre qui proteste contre les Girondins de retour: "si vous n'aviez pas attisé les brandons de la guerre civile départementale, jamais la Convention n'aurait été obligée de prendre des mesures extraordinaires ».

31. AN-D III-380, en date du 3 septembre 1793.

32. Sur les mésaventures du code civil, Jean-Louis HALPÉRIN, L'impossible code civil, Paris, 1992, p. 123-141.

33. La Convention le fait encore modifier le 31 août. Voir Hervé LEUWERs, op.cit., p. 78. Pour le texte définitif, $A P$, t. 73 , p. 246.

34. Les historiens - historiens de l'art inclus - ont longtemps interprété la participation enthousiaste des révolutionnaires aux lois de l'an II comme pure contrainte ou bien comme 
opportunisme. Il n'en est rien, ainsi que je l'ai démontré dans plusieurs contributions pour ce qui est des artistes, et ainsi qu'il en ressort des recherches ici entreprises.

35. Voir aussi Hervé LEUWERs, op.cit., p. 69-94. Nous ajoutons ici quelques détails non mentionnés par Leuwers, lesquels confirment néanmoins son interprétation. A savoir que personne n'a contraint Merlin à proposer de telles lois. La période qui suit ne fait que corroborer cette interprétation.

36. Le 23 thermidor an II, contre les opposants, Merlin décrit le tribunal révolutionnaire comme « le fruit des délibérations des trois comités réunis pendant un jour et trois nuits entières. On ne peut pas dire que ce soit là une loi extorquée ». Le Moniteur, t. 21, p. 448.

37. Le Moniteur, t. 21, p.369. Il fulminait aussi qu'on veuille désorganiser «totalement le gouvernement révolutionnaire ».

38. L'argument des circonstances ne date donc pas de Thermidor ou des historiens prorévolutionnaires du XIX siècle. Cambacérès l'évoque dès mars 1793 et persiste après Thermidor. A force de nier le rôle des circonstances, on en vient du reste à ne plus comprendre ce qui motivait ces hommes - ou à imputer leurs véritables motivations à une idéologie fixe, anachronique ou fantaisiste.

39. Le Moniteur, t. 21, p. 658 (séance du 16 fructidor an II).

40. Le Moniteur, t. 21, p. 473 (séance du 23 thermidor).

41. AN- D III* 56.

42. Séance du 25 frimaire an III. Le Moniteur, t. 22, p. 770.

43. Robert Lindet au peuple français, Paris, 1794, p. 7-8. Second mémoire de Robert Lindet, s.d., s.l.

44. Le Moniteur, t. 19, p. 14 et t. 18, p. 35 (rapport de Merlin sur l'ajout d'un cinquième juge en cas de désaccord des juges durant un procès et suppression d'une loi antérieure comme quoi, en cas de désaccord, c'était l'avis le plus doux qui prévalait).

45. AN D III-381 et D III * 56. La discussion du rapport de Merlin eut lieu au Comité de législation le 12 floréal an II. Le décret fut rendu le 19 floréal. Il réglait donc la compétence du tribunal révolutionnaire de Paris et celle des tribunaux criminels en France. Le Moniteur, t. 20, p. 419.

46. Diverses pièces dans AN F 7-4435. Sur les signataires de l'arrêté, voir Françoise BRUNEL, op. cit., p. 66.

47. Le Moniteur, t. 21, p. 741, 25 fructidor an II.

48. Le Moniteur, t. 24, p. 211-212, voir Hervé LEUWERs, op. cit., p. 92.

49. Par rapport évidemment à la loi des suspects qu'il avait lui-même conçue. Le Moniteur, t. 23, p. 77-79 et p. 105-110.

50. Les mémoires et défenses des membres de l'ancien Comité de salut public accusaient Robespierre d'avoir fait arrêter des individus selon son bon vouloir - et sous le sceau du Bureau de police générale. Les recherches d'Arne ORDING, Le Bureau de police du Comité de Salut public. Etude sur la Terreur, Oslo, 1931, ne permettent pas de conclure en ce sens. Jusqu'ici, nos propres recherches ont été peu concluantes sur ce point précis.

51. Notons toutefois que son intervention du 23 prairial en faveur de l'introduction d'un considérant ne portait pas sur ces points, mais sur les prérogatives du Comité de salut public au détriment de la Convention. Le 8 nivôse an III, en conséquence, Merlin dévoile tout à la fois ce qu'il pensait vraiment et ce qui était devenu lieu commun depuis Thermidor. Le Moniteur, t. 23, p. $105-110$.

52. Bulletin des lois, $\mathrm{n}^{\circ} 201$ et $\mathrm{n}^{\circ} 202$.

53. Ainsi était notamment supprimée la loi du 15 germinal sur la mise hors des débats. Voir aussi Bronislaw BACZKo, Comment sortir de la Terreur, p. 96 ff. Mais Baczko ne voit pas que le tribunal ne fut pas vraiment réformé le 23 thermidor et qu'il fut simplement réintroduit sous la forme qu'il avait avant le 22 prairial. La véritable réforme date du 8 nivôse an III et la suppression est du 12 prairial suivant. Entre-temps avait été introduite la loi sur la police générale du $1^{\mathrm{er}}$ germinal. 
Pour les dates correctes, voir Hervé LEUWERs, op.cit., p. 89. Pour le texte original, Bulletin des lois, $\mathrm{n}$ - 537, p. 4-16.

54. Encore Delacroix avait-il protesté que l'article sur la dépravation des mœurs lui paraissait incongru et pouvant mener à l'arbitraire. Les autres opposants à la loi s'étaient focalisés sur l'article XX. Le Moniteur, t. 20, p. 697-698 et p. 714.

55. Font défaut en effet les procès-verbaux qui recouvrent la période des 17 messidor-4 thermidor, ceux des 6-23 thermidor, et ceux enfin des 24 thermidor-16 fructidor. Voir AN D III* 56. Nos connaissances ne sont donc que fragmentaires, ce dont se plaignait déjà Georges Bourgin, qui donne néanmoins suffisamment d'informations bibliographiques.

56. Sur les nominations successives de Merlin, voir Hervé LEUWERs, op. cit., p. 85. Merlin revient au Comité de législation en nivôse an III.

57. AN D III*-58. La série AN D III*-57 contient le répertoire des personnes concernées, et non des procès-verbaux.

58. Le Moniteur, t. 23, p. 165.

59. Le Moniteur, t. 23, p. 156-157. Merlin y incrimine l'arrêté du 21 floréal et démontre que Maignet n'a fait que suivre les ordres. Ce dernier ne fut donc pas inquiété à ce moment-là.

60. On peut consulter les défenses des divers conventionnels et les procès qui leur sont faits à partir du 13 fructidor an II dans la série AD XVIII C 244-250.

61. Une exception cependant est Barras, qui mentionne mais sans spécifier les «dispositions draconiennes » de Merlin. Barras, Mémoires de Barras, 4 vols., Paris, 1895-1896, III, p. 393.

62. Contrairement à ce qu'écrit Baczko (et avant lui nombre d'historiens), il y a une solidarité remarquable entre les membres des comités, qui ne se trahiront jamais. A force d'être traînés dans la boue, ils dénonceront certes ceux qui en sont la cause et qui n'avaient aucune raison d'être aussi vindicatifs: Fréron et Tallien, en particulier. Pour les mémoires en faveur des accusés, AN AD XVIIIC-248 (Prieur de la Côte d'Or, Carnot, Lindet, Maure et Faure).Voir aussi les défenses de Barère, AN AD XVIII C-249. Un beau livre qui rend justice aux Conventionnels, celui de Sergio LuzzATTO, Mémoire de la Terreur, Lyon, PUL, 1991.

63. Le nom initial de cette commission était le comité de recensement et de classification des lois. Mais très vite, on parla de commission de classification. Voir les rapports de Rondonneau, AN D III 380.

64. Georges Bourgin regrettait justement que personne n'ait fait des recherches sur ces travaux de classification des lois. Carla Hesse dans l'article cité évoque sans plus cette commission, alors que cette dernière justement devait mettre fin à ce que Hesse nomme "une incessante prolifération » des lois.

65. Le 3 floréal an II, Couthon avait fait décréter l'établissement de deux commissions. L'une serait chargée de présenter un cours d'institutions sociales et l'autre un code des lois rendues jusque-là. La première était confiée au Comité de salut public; la seconde à Merlin, Cambacérès et Couthon. AP, t. 89, p. 169.

66. Est-ce si étonnant, quand on sait que Saint-Just se plaignait tout à la fois de l'absence d'institutions républicaines et des négligences des fonctionnaires. Mais l'article se trouvait mêlé à des dispositions pénales; c'est ce qui en fait l'étrangeté. Les plaintes contre l'inexécution des lois datent du mois d'août 1793. Voir AP, t. 73, p.171. Sur les projets de Saint-Just et BillaudVarenne, voir Françoise BRUNEL, op. cit., p. 46-52.

67. SAINT-JUST, Euvres complètes, Paris, 2004, p. 767. Le décret est du 27 germinal an II. Il sera amendé le 11 prairial sur proposition de Couthon, AP, t. 91, p. 147-148.

68. AN D-III 380.

69. AN D-III 380. Sont mentionnés les deux premiers codes (Constitution et Gouvernement révolutionnaire); ceux qui devaient inclure l'action du gouvernement: le code des relations extérieures, le code de police, de la justice criminelle, de la justice civile; des administrations civiles; de l'agriculture ; des dépenses et entrées ; du commerce ; des approvisionnements ; des 
ponts et chaussées ; des transports, postes, etc. ; le code monétaire et celui des secours publics. Pour ce qui était des moyens ou de la force, il y avait : le code de l'enseignement public; le code militaire; de la marine et des colonies; de l'armement; des fortifications; des domaines nationaux; des eaux et forêts ; des contributions ; de la trésorerie nationale, de la liquidation et de la comptabilité. Font défaut sur cette liste celui de l'émigration et celui des sciences exactes. Voir aussi la liste donnée par Georges Bourgin, op. cit., p. 636, qui, curieusement, ne mentionne pas la série D- XXXIX, qui contient les minutes du code rédigées par Rondonneau et celles du comité de la classification des lois.

70. Ce rapport du 27 messidor an II est publié dans Mémoires inédits de Cambacérès, 2 vols., Perrin, 1999, I, p. 219-227.

71. Ibidem.

72. AN D III-380. D III-362.

73. Louis Rondonneau est devenu le propriétaire et le fondateur du dépôt des lois. Sous l'Empire, il est notamment bibliothécaire au Conseil d'Etat. On lui doit enfin plusieurs éditions de la Collection générale des lois, décrets, arrêtés, senatus consultes publiés depuis 1789, de même qu'une Table générale alphabétique et raisonnée des matières contenues dans le Répertoire de jurisprudence et dans le recueil alphabétique des questions de droit de M. Merlin, Paris, 1829. Cette table donne une idée de la façon méthodique dont il entendait son travail. Ses contacts avec Merlin semblent avoir été excellents, ainsi qu'en témoigne une lettre de ce dernier du 13 avril 1820 publiée dans la Table susnommée. Sur Rondonneau, voir l'étude détaillée de N. Choublier-Grimben, «Louis Rondonneau et le Dépôt des lois ", Bibliothèque de l'Ecole des Chartes, t.166, 2008, p. 195-240. L'auteur n'aborde pas la période conventionnelle.

74. Lors d'une autre séance, il était prévu que Rondonneau rende compte chaque jour à la Commission. AN D-III 380. A lire les consignes données aux fonctionnaires et à voir leur nombre, on assiste alors à une véritable bureaucratisation de la fonction publique.

75. Cambacérès, Mémoires, I, p. 223.

76. Le Moniteur, t. 22, p. 658.

77. Le Moniteur, t. 24, p. 33-37. Ainsi, comme sous la Constituante venait en premier la répression au nom de l'ordre public. La même chose vaut pour le Directoire dont le Code des délits et des peines est le premier à voir le jour. Bulletin des lois, no.1221 du 3 brumaire an IV - avec la réorganisation de l'instruction publique (même date).

78. Sur le code révolutionnaire, AN D-XXXIX - 9 et 10.

79. Après le 9 Thermidor, on a vu qu'il n'était nullement question de le supprimer - il se perpétrerait jusqu'à la paix générale - ou du moins jusqu'à l'entrée dans le régime constitutionnel. Le tribunal par contre est supprimé le 12 prairial an III, mais la commission militaire qui s'y substitue dès le 4 prairial n'est sans doute pas plus clémente. Sur la justice exceptionnelle de l'après-Thermidor, Howard G. BROWN, Ending the French Revolution. Violence, Justice and Repression from the Terror to Napoleon, University of Virginia Press, 2006.

80. Cela donnera lieu à la loi du 14 frimaire an II, qui décrétait notamment la création d'un Bulletin des lois de la République et le remplacement des procureurs syndics par des agents nationaux.

81. Voir D-III 321, 1. Sur les juges de paix qui demandent qu'on leur envoie les lois - qu'ils n'ont donc pas - et sur la question de savoir si l'agent national a le droit ou non d'apposer les scellés. Qui plus est, le Comité des pétitions faisait souvent parvenir sa propre correspondance au Comité de législation.

82. AN D-III* 54-56.

83. AN D-XXXIX-10.

84. AN D XXXIX-9. Ce texte hautement idéologique pourrait être de Billaud-Varenne à qui Rondonneau semble avoir envoyé les premiers titres. Rondonneau ne se permettait pas de tels apartés. Ses remarques à lui sont strictement juridiques. 
85. AN D XXXIX-9.

86. Le problème des taxes abusives sera soulevé après Thermidor. Notamment à propos de la mission de Fouché dans l'Allier. AN D-III 348.

87. Voir dans ce numéro, l'article de Raphaël Matta. Sur le Comité de sûreté générale, voir celui d'Emilie Cadio.

88. AN D XXXIX-10. Ce carton - microfilm - comprend les deux premières parties du code révolutionnaire.

89. AN-D III-381.

90. C'est si vrai que le 22 fructidor an 2, le Comité de législation donne encore et toujours la priorité à la surveillance des corps constitués. AN D-III* 58 .

91. En principe, car dans la réalité, il y eut donc des excès. On y reviendra dans un article ultérieur. Inversement, nombre de représentants en mission ont été fidèles à leur mission. Voir Michel BIARD, Missionnaires de la République. Les représentants du peuple en mission (1793-1795), Paris, CTHS, 2002.

92. Le Moniteur, t. 21, p. 687-688.

93. Les premières lois contre les émigrés sont en réalité du 9 juillet 1791 et du 9 novembre de la même année. Elles s'aggravent le 9 février 1792, ensuite le 27 juillet (confiscation de biens). Le $1^{\text {er }}$ mars 1793, la Convention les déclare « bannis à perpétuité » et « morts civilement ».

94. AN DXXXIX- 10.

95. Il fallut pourtant bien en exporter pour payer les subsistances et les produits de base dont avait besoin la Convention pour survivre. C'est ainsi que furent exportés en Amérique des produits de luxe, saisis dans les maisons "nationales" pour rembourser les commandes de grains, de potasse, de salpêtre, de suif, de cuivre et autres métaux ou matériaux. F 11-223 et F 11-292-293. Toute une équipe d'Américains trafiquait pour le compte de la France. Sur cet aspect de la Révolution, notre article à paraître, « Business and Liberty. Four patriots during the Atlantic Revolution ", Congress of Dunfee, University of New Hampshire (à paraître).

96. Vivant Denon en témoigne dans ses Lettres à Bettine, où il évoque les tours de garde qui lui sont imposés, ce qui ne l'enchante guère. Ailleurs, un rentier se plaint des réquisitions en argent que revendique continuellement sa section. Ailleurs encore, une femme se voit contrainte de verser 3000 livres sous peine d'être poursuivie. Il est certain aussi que certains comités de surveillance abusaient de leurs pouvoirs. Vivant Denon, Lettres à Bettine, Actes Sud, 1999, p. 285-299 et Célestin GUITTARD, Journal d'un bourgeois de Paris sous la Révolution, Paris, 1974, p. 260 et p. 285-286. Voir aussi AN F7-4435.

97. AN DXXXIX-11. Ces réquisitions diverses, et pas forcément militaires, sont incluses dans le code militaire.

98. La loi recensée est celle du 17 septembre, suivie de tous les décrets ultérieurs concernant des cas particuliers, tels que les marchands qui ne respecteraient pas le maximum; les tanneurs qui ne vendent pas et ne remplissent pas leurs postes sans interruption ou les détenteurs de biens nationaux qui n'auraient pas fait leur déclaration. L'article 1 précise : les gens suspects sont en état d'arrestation. Les suspects étaient conduits dans les maisons d'arrêt du lieu de leur détention. Que je sache, la loi du 22 prairial n'est pas incluse. Ce peut être une question de date. Rondonneau en tout cas en a définitivement effacé le souvenir dans sa Table générale alphabétique et raisonnée sur les lois depuis 1789.

99. AN D XXXIX-10.

100. Deux lois des 25 floréal et 12 prairial an II prévoyaient que le Comité de salut public contrôle les jugements des commissions populaires et examine la liste des condamnés à mort et des acquittés depuis la création du Tribunal révolutionnaire. AN F7-4436/1, vol. 3, pièce 19. Les 2 et 3 thermidor, deux nouveaux arrêtés spécifiaient que les prévenus jugés par les commissions populaires seraient immédiatement mis en jugement - après que les deux comités du gouvernement avaient paraphé leur décision. Recueil des Actes du Comité de Salut public, t. 15, p. 316 
et p. 334. Voir aussi AN F7-4599. Sur les avis des commissions populaires, voir les pièces imprimées dans le rapport de Saladin, Rapport au nom de la Commission des Vingt-Un fait le 12 ventôse par Saladin, Paris, ventôse an III, AN AD XVIIIC-248. Le plus souvent, elles conseillaient la déportation.

101. Voir les détails dans Réponse des membres de l'ancien comité de salut public dénoncés aux pièces communiquées par la Commission des vingt-un, Paris, ventôse an III, p. 79-90. AN AD XVIIIC-249.

102. C'est ce qui sera reproché aux membres de l'ancien Comité de salut public. On jugea même que ses dispositions exceptionnelles (le seul verdict étant la mort et les preuves étant la conviction intime), annonçaient la loi du 22 prairial, ce qui paraissait confirmer la culpabilité des trois grands " coupables ». Attribué à Robespierre, l'arrêté initial pourtant était signé des autres membres du Comité de salut public. Voir Françoise BRUNEL, op. cit., p. 65-66.

103. AN F7- 4436/1, pièce 243. Sur les condamnations peu "orthodoxes" d'Orange, voir Françoise BRUNEL, Ibidem, p. 66. D'après l'enquête d'Anne-Marie Duport citée par Françoise Brunel, sur les 432 condamnations prononcées, une centaine portait des peines de réclusion et des amendes. Les commissions populaires, du reste, proposaient pour la plupart des peines de déportation, et non des exécutions. Sur les réalités judiciaires, Robert ALLEN, Les tribunaux criminels sous la Révolution et l'Empire, Rennes, PUR, 2005.

104. Au sujet des abus ou des erreurs, voir notamment les explications des anciens membres du Comité de salut public, AN AD XVIIIC-249.

105. Un nouveau rapport fut fait le 8 messidor, concernant la loi relative à la formation d'un livre de Bienfaisance nationale, laquelle concernait les cultivateurs et artisans vieillards et infirmes, ou les femmes démunies des campagnes. Le premier décret était du 22 floréal. Procès-verbaux de la Convention, t. 37, p. 124, et pour le 8 messidor, ibid., t. 63, p. 115-117. Voir Françoise BRUNEL, op. cit., p. 59-60.

106. Le 19 prairial, une loi prévoit également de distribuer des secours publics aux réfugiés étrangers - Liégeois, Belges, Mayençais et tous ceux qui souffraient de l'occupation des armées ennemies. P.V. de la Convention, t. 39, p. 108-109.

107. AN DXXXIX -11. Le Comité de salut public arrêta en effet une loi pour surveiller et accélérer la confection des listes.

108. Sur les problèmes rencontrés pour mener à bien cette œuvre, Françoise BRUNEL, op.cit., p. $74-75$.

109. Le 8 germinal an III, alors qu'il est encore question d'achever les lois organiques de la Constitution de 1793, Merlin de Thionville et Merlin de Douai convenaient cependant que le code judiciaire était quasiment terminé. De fait, il sera voté le 3 brumaire an IV. Le Moniteur, t. 24, p. 90-91. Il ne restait plus, disaient-ils, qu'à légiférer sur le pouvoir exécutif. L'abandon définitif de cette constitution date des journées de Germinal et de Prairial.

110. En l'an IV, la liquidation des dettes des émigrés n'était pas encore achevée. AN DXXXIX- 12.

111. AN DXXXIX-11.

112. AN DXLII-7, pièce 103.

113. Le Moniteur, t. 17, p. 680 (17 septembre 1793).

114. Nous ne mentionnons pas ici l'instruction publique, mais on le sait, celle-ci était évidemment au centre des préoccupations. Le décret du 29 frimaire an II y était consacré, et 6831 écoles avaient déjà été créées avant Thermidor. Bernard LEHEMBre, Naissance de l'école moderne. Textes fondamentaux, Nathan, 1989, p. 118.

115. La législation politique par contre était plus que succincte et entièrement sous sa domination. Furent réalisés un Code rural et forestier ; un Code militaire ; un Code de commerce parallèlement au Code civil, et plus tard au Code criminel. Rondonneau publia aussi un recueil sur les lois françaises introduites dans les pays annexés par Napoléon à partir de 1810. 
116. AN DXLII-7, pièce 102. Se pourrait-il que cette liste soit celle de la commission chargée du cours des institutions sociales, confié au Comité de salut public, et plus précisément à Saint-Just ? Voir AP, t. 89, p. 169.

117. La somme mentionnée de 600 millions paraît invraisemblable, d'autant que la guerre et les subsistances absorbaient la plupart des fonds dont disposait la Convention. Tout ce qui suit provient de DXLII-7, pièce 102.

118. Sur les difficultés rencontrées par plusieurs projets, Françoise BRUNEL, op. cit., p. 74-75.

119. Sur cette loi qui sera encore à l'ordre du jour le 8 messidor, Ibidem, p. 59-61. Le 22 floréal était créé le Livre de la Bienfaisance nationale. Pour vérifier si ces lois ont bien été décrétées, voir Procès-Verbaux de la Convention, Paris, an II, t. 37, t. 38 et t. 39 aux dates mentionnées.

120. On trouvera ces lois au titre 148 sur les indemnités dans le Répertoire des lois de G. Beaulac, op. cit., p. 539-540. La Convention est la seule à s'en être préoccupée à ce point. De même, elle a surpassé de loin les assemblées précédentes en ce qui concerne les lois ou décrets sur les sciences et les arts, p. 560-564, titre 157. Ces priorités civiles, sociales et culturelles nuancent donc l'interprétation trop univoque de Carla Hesse.

121. Le Comité des subsistances était fort actif. D'énormes sommes étaient en jeu, et le plus souvent, il fallait payer comptant. Les révolutionnaires avaient donc besoin de banquiers patriotes, même à l'étranger. En ventôse an II, par exemple, 125 millions de livres étaient nécessaires, alors que les banquiers n'en proposaient que 50. De germinal an II à vendémiaire an III, 850 millions avaient été mis à la disposition des subsistances. Voir les tableaux dans AN F11-292 et 293. C'est un aspect de la Révolution qui mériterait d'être mieux étudié. Néanmoins, Pierre CARON, Le Comité des subsistances de l'an II, Paris, 1924.

122. Sur la pleine réalisation des décrets de ventôse, il est difficile de conclure. Il semblerait que les autorités départementales aient renâclé à les exécuter, car ces administrations comprenaient de grands propriétaires. Couthon s'en plaignait aux Jacobins le $1^{\mathrm{er}}$ floréal. Collot ajoutait que « le plus grand avantage pour la république est de multiplier les propriétaires » - contre Couthon, en ce sens que, lui, était conscient que l'opération rapporterait peu à la France. Société des Jacobins, VI, p. 85-87.

123. Mss. No.144. Bibliothèque historique de la ville de Paris.

124. Jean-Clément martin, Violence et Révolution. Essai sur la naissance d'un mythe national, Paris, Seuil, 2006.

125. Et évidemment par la Constituante et la Législative qui avaient ouvert la voie, mais avaient eu moins d'obstacles à surmonter.

126. Les Thermidoriens parleront de 14 mois de dictature robespierriste. C'est évidemment bien moins long. Son pouvoir quasi absolu devient perceptible après la chute des factions, quand il impose à la Convention la loi sur l'Etre suprême et puis celle du 22 prairial. Notons par ailleurs que Robespierre semble avoir apprécié Merlin, ainsi qu'en témoignent ses propos du 24 prairial.

127. Par idéologues, nous pensons à Robespierre et Saint-Just, trop souvent mis en avant - pour le meilleur et pour le pire - dès qu'il s'agit de la Convention. Or, leur rôle paraît plutôt limité quand on examine la fabrique des lois. Nous avons voulu ici rendre leur véritable dimension aux personnages soi-disant secondaires, en raison de leur rôle éminent et durable dans la législation révolutionnaire.

128. C'est bien ce que reprocheront les Thermidoriens à la loi du 22 prairial, imposée par le «triumvirat » sans qu'elle ait été discutée par les personnes habilitées. De même il n'était pas rare qu'une loi soit rapportée ou modifiée, ce qui en rendait l'exécution encore plus difficile dans les départements.

129. Merlin se plaignait même le 26 septembre 1793 que les comités ne se soient pas encore réunis pour rédiger l'acte d'accusation. AN F7-4443, plaquette 4.

130. Que ce soit des remises en liberté ou des arrestations pour vices de procédure. AN D-III* 54 et 58 . 
131. Bien qu'il soit indéniable que le pénal eut et conserva la priorité, et ce même après Thermidor.

132. Les décrets sont légion à la Convention qui accordent telle ou telle somme aux victimes d'injustices. Le Comité de législation y contribuait. On peut comparer avec l'Amérique comme le fait Dominico LosURDo dans son dernier ouvrage provocateur, Liberalism. A Counter-History, Verso, 2011, où il suggère que les Etats-Unis ont peu légiféré en matière de lois civiles, préférant laisser aux Etats leurs prérogatives - et leurs égoïsmes.

133. Sur le sujet, notre article «Les discours sur la terreur à l'époque révolutionnaire (1776-1798). Etude comparative sur une notion ambiguë », French Historical Studies, nº 36, I, 2012. Rappelons que les régimes totalitaires et terroristes sont justement caractérisés par l'absence de lois positives. Hannah ARENDT, Le système totalitaire, Paris, 1972, p. 281-87 et ibid., Crises of the Republic, New York, 1969, p. 154-155.

134. Cet aperçu ébranle quelque peu les interprétations idéologiques fantaisistes, qui se fondent sur quelques discours subjectivement sélectionnés de Robespierre et de Saint-Just. Notamment, Dan EDELSTEIN, The Terror of Natural Right. Republicanism, the Cult of Nature and the French Revolution, Chicago, CUP, 2009.

\section{RÉSUMÉS}

La Révolution a énormément légiféré, c'est bien connu. Des milliers de lois ont alors été discutées et rédigées par le Comité de législation, qui touchaient à la justice pénale et criminelle mais aussi à la société civile et aux institutions républicaines. C'est de ce comité qu'il sera question ici, et en particulier, du comité sous la Convention et de son rôle dans les lois dites terroristes. S'y ajoutera un aperçu de ses autres missions, notamment celle qui concerne la classification et la simplification des lois, entreprises toutes deux sur ordre de la Convention de l'an II.

The period of the Convention has been one of the most studied and the most interpreted, but no research has been done on the whole body of legislation that it enforced. Too much importance has been attached to some of these laws - like the law of 22 Prairial - whereas others have been absolutely neglected. Furthermore, almost no attention has been given to the Legislation committee, although its members were the ones who proposed and drafted all these laws. Amongst them were two key lawyers, Merlin de Douai and Cambacérès, who were responsible for devising the civil and penal texts of this period - except for the law of 22 Prairial. Not only is it essential to know more about their proposals on this topic, but it is also salient to realize that the Convention was busy with the classification and simplification of all laws implemented since the very beginning of the Revolution. A commission was appointed to do the job. At its head was Louis Rondonneau, the great collector and publisher of revolutionary and imperial legislation until the 1830s.

Such an approach can be useful to help us gain a better understanding of the French Revolution. Indeed, it allows us to rediscover some secondary actors who are considered moderates although they were the authors of the most repressive laws of 1793. They even wanted these laws to be perpetuated after Thermidor. Moreover, such an approach gives us concrete proof that the Convention was also preoccupied by common (civil) laws - or what they called 'democratic laws'and not only by penal and criminal issues - or what has been called revolutionary government. 
And this revolutionary government was maintained until the enforcement of the 1795 Constitution.

INDEX

Mots-clés : législation, justice, code, lois démocratiques, étrangers, émigrés, suspects, tribunal révolutionnaire, gouvernement révolutionnaire, Cambacérès, Merlin de Douai

Keywords : Legislation, Justice, Civil Code, Criminal Code, Democratic laws, Foreigners, Emigrants, Suspects, Revolutionary Tribunal, Revolutionary Government, Cambacérès, Merlin de Douai

\section{AUTEUR}

\section{ANNIE JOURDAN}

Université d'Amsterdam 